\title{
On the Origin of Charge-Asymmetric Matter. I. Geometry of the Dirac Field
}

\author{
Alexander Makhlin \\ Rapid Research Inc., Southfield, MI, USA \\ Email: amakhlin@comcast.net
}

Received 25 February 2016; accepted 25 April 2016; published 28 April 2016

Copyright (C) 2016 by author and Scientific Research Publishing Inc.

This work is licensed under the Creative Commons Attribution International License (CC BY). http://creativecommons.org/licenses/by/4.0/

(c) (i) Open Access

\section{Abstract}

This work presents new round of the author's pursuit for consistent description of the finite sized objects in classical and quantum field theory. Current paper lays out an adequate mathematical background for this quest. A novel framework of the matter-induced physical affine geometry is developed. Within this framework, (1) an intrinsic nonlinearity of the Dirac equation becomes self-explanatory; (2) the spherical symmetry of an isolated localized object is of dynamic origin; (3) the auto-localization is a trivial consequence of nonlinearity and wave nature of the Dirac field; (4) localized objects are split into two major categories that are clearly associated with the positive and negative charges; (5) of these, only the former can be stable as isolated objects, which explains the global charge asymmetry of the matter observed in Nature. In the second paper, the nonlinear Dirac equation is written down explicitly. It is solved in one-body approximation (in absence of external fields). Its two analytic solutions unequivocally are positive (stable) and negative (unstable) isolated charges. From the author's current perspective, the so for obtained results must be developed further and applied to various practical and fundamental problems in particle and nuclear physics, and also in cosmology.

\section{Keywords}

Dirac Field, Affine Geometry, Localization, Cosmological Charge Asymmetry

\section{Introduction}

This work addresses the long-standing puzzle of how the physical Dirac field of real matter becomes a finitesized particle. This puzzle successfully withstood several major attacks undertaken since early 1930s in both classical and quantum contexts, for realistic fields of matter and for the ad hoc constructed effective field theories. The importance of a definitive conclusion goes far beyond the purely academic area. The present 
uncertainty of an answer affects numerous studies in theoretical and experimental physics (e.g., quantum collisions of finite-size ultrarelativistic nuclei [1]) and reaches as far as the origin of the observable matter in the Universe. A dramatic difference between the charge asymmetry of the visible Universe and an apparent charge symmetry observed in transformations of elementary particles has never received a rational explanation. In mid1960s, A.D. Sakharov [2] made an attempt to connect the cosmological charge asymmetry with the violation of the $C P$-invariance and nonequilibrium processes in the early hot Universe, but this hypothesis cannot be verified by a laboratory experiment. More specified (and exotic) scenarios were considered by A. Dolgov [3] [4], partially in connection with the problem of baryogenesis. For an extensive review with further references see Ref. [5].

The present study concludes that, for the Dirac field, $C$ and $P$ do not exist separately, and that both are intimately connected to inevitable localization of the Dirac field into finite-sized particles. Furthermore, it appears that only positive charges are capable of stable auto-localization in real world. The time scale and relative weight of all the underlying processes and/or mechanisms are not yet clear, but the Universe definitely had enough time to conduct such an experiment. Moreover, experimental studies of the last decade [6] revealed a surprising excess of positrons (and no excess of antiprotons) in the cosmic rays, which can be an indication that creation of the charge-asymmetric matter in the Universe is an ongoing process.

The present work was supposed to correct and augment the author's paper [7], which was focused mainly on the transient processes with localized particles. The accents have changed with the initial progress. In this work and then in paper [8], we pursue a somewhat narrower goal to find an exact auto-localized solution (a realistic Dirac particle), which could serve as an input for the study of transient processes. The problem is posed and solved in a novel framework of the matter-induced affine geometry, which deduces geometric relations in the space-time continuum from the dynamic properties of the Dirac field.

Framework is set in Section 2 by reviewing well-known algebraic identities between the bilinear Dirac forms (the Fierz identities). At any point in spacetime continuum (the principal differentiable manifold $\mathbb{M}$ ), there exist four fields of quadruples of these forms (the Dirac currents), which are linearly independent and Lorentzorthogonal, and can serve as local algebraic basis for any four-dimensional vector space, including the infinitesimal displacements in coordinate space $\mathbb{R}^{4}$.

In Section 3 we use this basis of four Dirac currents as the Cartan's moving frame in spacetime and develop the technique of covariant derivatives for the vector and spinor fields.

Relying on results of Section 2 and Section 3, we meticulously derive in Section 4 various differential identities from the Dirac equations of motion. These identities are shown to be imperative for the geometry of the objects associated with the Dirac field to have a covariant form and be independent of coordinate background. We discover that coordinate lines and surfaces cannot be chosen by a fiat-the Dirac field cannot be embedded into a coordinate basis $\left(\partial / \partial x^{\mu}\right)$ (this observation had triggered the present work starting from [7], where the key argument regarding localization was found). In Section 5 the differential identities for the divergences and curls of the Dirac currents are written down in terms of components, and properties of the congruences of the Dirac currents are analyzed. All components of the connections are found as functions of the Dirac field. These two steps finalize the formal design of the physical affine geometry. There are only a few digressions regarding physical meaning of some equations, the most important of which is related to the existence of the matterdefined world time $\tau$ and the local time slowdown. The latter is the main physical mechanism behind the autolocalization. It appears that, in order to be compatible with the Dirac equation, its coordinate basis indeed cannot be holonomic.

The known connections made it possible to examine the properties of the admissible coordinate systems. Among four tetrad vector fields, we find in Section 6.1 two integrable subsets of three PDEs for the coordinate lines (two hypersurfaces with the corresponding normal congruences) and two two-dimensional surfaces. In Section 6.2 we study the internal geometry of these surfaces as submanifolds of $\mathbb{M}$. It appears that the twodimensional surface of the constant "world time" and "radius" can be only spherical, which seems to be inevitable for an isolated stable object.

The general properties of coordinate surfaces in $\mathbb{M}$ (like their spherical symmetry and inherent stability) are discovered in the present paper without any assumptions on the nature of an ambient space or Dirac field. It appears that the main qualitative characteristic of the stationary Dirac object is the direction of the axial current, which can point only outward or inward. It must be clearly understood that the locally defined notions of outward and inward are prerequisites for any reasonable discussion of the localization phenomenon. The frame- 
work of the matter-induced affine geometry not only ideally fits this goal but also explains the auto-localization, as it is seen in the real world, as an intrinsic property of the Dirac field.

This paper is continued in Ref. [8], where the capabilities of the matter-induced affine geometry are employed to address a specific problem of existence of the auto-localized Dirac waveforms. We begin with writing down the nonlinear Dirac equation and putting it in a practically solvable form. The localized configurations of the Dirac field are found analytically in the absence of external electromagnetic field. They require the Dirac spinor to have only up- or only down-components, when the axial current is pointing outward or inward, respectively. The up-mode is stable, has a bump of invariant density and the negative energy $E=-m$, while the down-mode is unstable, has a dip and the positive energy $E=+m$. At large spatial distances the invariant density has a universal vacuum unity value. Therefore, the two modes were (by a fortunate coincidence!) properly interpreted as positive and negative charges. The decay of unstable mode is due to the charged Dirac currents that naturally oscillate as $\mathrm{e}^{2 i m \tau}$, such a decay requiring only the presence of an external electromagnetic field. Possibly, these facts explain the vivid global charge (eventually, baryonic one) asymmetry in the Universe. Last section of paper [8] summarizes ideas, methods, current results and perspectives.

\section{Vectors at a Point. Algebra of the Dirac Currents}

1. Mathematical framework. We consider, as usually, the mathematical spacetime as a smooth fourdimensional manifold $\mathbb{M}$ so that every point $\mathrm{P}$ of $\mathbb{M}$ has an open neighborhood that can be mapped one-toone onto an opened subset of points $\left(x^{0}, x^{1}, x^{2}, x^{3}\right) \in \mathbb{R}^{4}$. From the viewpoint of the differential topology, one has to start with scalar functions $f(\mathrm{P}(\lambda)) \subset \mathbb{M}$ on the curves $\mathrm{P}(\lambda)$ (determined by a map $\mathbb{R}^{1} \rightarrow \mathbb{M}$, $\left.\lambda \in \mathbb{R}^{1}\right)$ in order to build at each point $\mathrm{P} \in \mathbb{M}$ the linear space $T_{p}(\mathbb{M})$ of tangent 4-vectors

$$
\boldsymbol{h}_{\lambda}(\mathrm{P})=(\mathrm{d} / \mathrm{d} \lambda)=\partial_{\boldsymbol{h}}=\left.\left(h^{\mu} \partial_{\mu}\right)\right|_{\mathrm{P}(\lambda)}
$$

with the components $h^{\mu}$ with respect to the linearly independent vectors $\left(\partial / \partial x^{\mu}\right)_{\mathrm{P}}$ of the coordinate basis in $\mathbb{R}^{4}$.

Being defined via the mapping $\mathbb{R}^{1} \rightarrow \mathbb{M}$, a curve and its tangent vectors are invariant objects; only the components $h^{\mu}$ of a vector explicitly depend on a particular choice of coordinates in $\mathbb{R}^{4}$. Action of operator (2.1) on the functions $f(x)=x^{v}$ yields the system of ODEs for the unknown $x^{\mu}, \mathrm{d} x^{v} / \mathrm{d} \lambda=h^{v}(x)$. It is said that $u^{\mu}$ are components of a vector if they are transformed as components $\mathrm{d} x^{\mu}$ of a displacement $\mathrm{d} x$.

Any four linearly independent vectors $\boldsymbol{h}_{a}=\mathrm{d} / \mathrm{d} \lambda^{a}=h_{a}^{\mu} \partial_{\mu}, a=0,1,2,3$, (with the non-degenerate matrix $\left|h_{a}^{\mu}\right|$, det $\left|h_{a}^{\mu}\right| \neq 0$ ) can be used as the basis. Then there also exists the inverse matrix $h_{\mu}^{a}$ of the 1-forms $\boldsymbol{h}^{a}$ so that $h_{a}^{\mu} h_{v}^{a}=\delta_{v}^{\mu}$ and $h_{a}^{\mu} h_{\mu}^{b}=\delta_{a}^{b}$. Since any quadruple $\boldsymbol{u}$ of numbers can be expanded over the basis $\boldsymbol{h}_{a}$, we have $\boldsymbol{u}=u^{a} \boldsymbol{h}_{a}=u^{a} h_{a}^{\mu} \partial_{\mu}=u^{\mu} \partial_{\mu}$. Therefore, $u^{a}=\boldsymbol{u} \boldsymbol{h}^{a}=h_{\mu}^{a} u^{\mu}$ and $\mathrm{d} \lambda^{a}=h_{\nu}^{a} \mathrm{~d} x^{v}$, but in general, $\mathrm{d} \lambda^{a}$ are not the total differentials of any independent variables.

2. Physical framework. Basis of Dirac currents. In physical spacetime of special relativity points $P$ are associated with events. The clocks of the net that register these events are synchronized by light signals; this results in Lorentz transformations between the coordinates of events measured by the nets of different inertial observers. Special relativity is based on independence of all physical processes from a particular choice of an inertial frame, and thus from the coordinate basis that is used to parameterize the events. As a matter of fact, the coordinate basis is built into a material reference frame, and thus is an invariant object.

All mathematical treatments of affine or Riemannian geometry start with an assumption of the independent tangent space with an arbitrarily oriented normal basis at every point of the continuum (differentiable manifold). While invariance with respect to the choice of coordinates $x^{\mu}$ is trivial, there cannot be absolute freedom of choosing tetrad vectors at every point-the components $h_{a}^{\mu}(x)$ of tetrad vectors must be continuous functions of the coordinates. Is there a way to endow the principal manifold $\mathbb{M}$ with basis of vector fields that would be invariant objects without reference to curves and/or derivatives at a point? For the physical four-dimensional spacetime the answer is affirmative, because there exists a matter field, the Dirac field $\psi(\mathrm{P})$, a coordinate scalar, that provides such a basis at each point $\mathrm{P}$ of the manifold $\mathbb{M}$ and assigns the latter the status of a physical object. The algebraic descendants of the Dirac field are the vector-like objects, the so-called Dirac currents,

$$
\boldsymbol{J}_{[0]}(\mathrm{P})=j^{a}(\mathrm{P})=\psi^{+} \alpha^{a} \psi, \quad \boldsymbol{J}_{[3]}(\mathrm{P})=J^{a}(\mathrm{P})=\psi^{+} \rho_{3} \alpha^{a} \psi, \quad \boldsymbol{J}_{[1]}(\mathrm{P})=\Theta^{a}(\mathrm{P}), \quad \boldsymbol{J}_{[2]}(\mathrm{P})=\Phi^{a}(\mathrm{P}),
$$


of which the last two are the real and imaginary parts of the complex "matrix element" between the two chargeconjugated configurations, $\Lambda_{(+)}^{a}=\left(\psi_{c}\right)^{+} \alpha^{a} \psi=\Theta^{a}+i \Phi^{a}$ and $\Lambda_{(-)}^{a}=(\psi)^{+} \alpha^{a} \psi_{c}=\Theta^{a}-i \Phi^{a}$, where $\psi_{c}$ is the charge-conjugate spinor.

The components $J_{A}^{a}$ of the currents $\boldsymbol{J}_{(A)}(\mathrm{P})$ depend only on the Dirac field and on a particular choice of the matrices $\alpha^{a}$ at the point $\mathrm{P}$. The numbers $\boldsymbol{J}_{[0]}(\mathrm{P})$ are the coordinate scalars but are dubbed components of the "vector current". Another four real numbers, $\boldsymbol{J}_{[3]}(\mathrm{P})$, are associated with the components of the "axial current". The idea to use $\Theta^{a} / \mathcal{R}$ and $\Phi^{a} / \mathcal{R}$ as the tetrad vectors was first spelled out in Ref. [9].

In these definitions, an explicit form of the Dirac matrices $\alpha^{a}=\left(1, \alpha^{i}\right)=\left(1, \rho_{3} \sigma^{i}\right), \rho_{i}$ and $\sigma^{i} \quad(a=0,1,2$, 3 ; $i=1,2,3)$, is not specified; it is only required that they satisfy commutation relations,

$$
\alpha^{a} \beta \alpha^{b}+\alpha^{b} \beta \alpha^{a}=2 \beta \eta^{a b}, \alpha^{a} \beta+\beta \alpha^{a}=0,
$$

and, in general, they are not just numeric matrices. One can resort to a particular set of numerical matrices $\alpha^{a}$ and $\beta$ only in conjunction with the corresponding tetrad basis $\boldsymbol{h}_{a}{ }^{1}$.

3. Fierz identities. Completeness of the basis. It appears that the four quadruples, $\boldsymbol{J}_{A}(\mathrm{P})(A=0,1,2,3)$, along with the scalar $\mathcal{S}$ and pseudoscalar $\mathcal{P}$, satisfy the following identities ${ }^{2}$,

$$
\begin{aligned}
& \mathcal{R}^{2} \equiv j_{a} j^{a}=-\mathcal{J}_{a} \mathcal{J}^{a}=-\Theta_{a} \Theta^{a}=-\Phi_{a} \Phi^{a}=\mathcal{S}^{2}+\mathcal{P}^{2}, \\
& j^{a} j^{b}-\mathcal{J}^{a} \mathcal{J}^{b}-\Theta^{a} \Theta^{b}-\Phi^{a} \Phi^{b}=\mathcal{R}^{2} \eta^{a b}, \\
& j_{a} \mathcal{J}^{a}=j_{a} \Theta^{a}=j_{a} \Phi^{a}=\mathcal{J}_{a} \Theta^{a}=\mathcal{J}_{a} \Phi^{a}=\Theta_{a} \Phi^{a}=0,
\end{aligned}
$$

where $\eta_{a b}=\operatorname{diag}(1,-1,-1,-1)$ is the Minkowski tensor (which was not contemplated to be here) and $j_{a}=\eta_{a b} j^{b}, \ldots$ The Dirac currents $\boldsymbol{J}_{A}(\mathrm{P})$ are almost always linearly independent ${ }^{3}$. In what follows, unless stated otherwise, we will consider only "regular" domains where $\mathcal{R}^{2}>0$ and use, instead of $\boldsymbol{J}_{(A)}$, the normalized currents $\boldsymbol{V}_{A}=\boldsymbol{J}_{A} / \mathcal{R}$. The matrix $V_{A}^{a}$ is not degenerate and thus has an inverse matrix $V_{a}^{A}$,

$$
V_{A}^{a} V_{a}^{B}=\delta_{A}^{B}, V_{A}^{a} V_{b}^{A}=\delta_{b}^{a} .
$$

By virtue of Equation (2.3), at every point $\mathrm{P}$ of the basic manifold $\mathbb{M}$ the currents $\boldsymbol{V}_{A}$ form a complete (in the sense of linear algebra) system of orthogonal (with respect to the " metric" $\eta_{a b}$ ) unit "vectors",

$$
\eta_{a b} V_{A}^{a} V_{B}^{b}=\eta_{A B}, \quad \eta^{A B} V_{A}^{a} V_{B}^{b}=\eta^{a b} .
$$

The vector $V_{0}$ is timelike while the other three are spacelike. It is also straightforward to check the following identities,

$$
V_{A}^{a}=\eta^{a b} \eta_{A B} V_{b}^{B}, V_{a}^{A}=\eta_{a b} \eta^{A B} V_{B}^{b},
$$

${ }^{1}$ Employing the Dirac matrices, we can define the four components of the "vector current", $j^{a}=\psi^{+} \alpha^{a} \psi \equiv \bar{\psi} \gamma^{a} \psi$, the four components of the "axial current", $J^{a}=\psi^{+} \rho_{3} \alpha^{a} \psi \equiv \bar{\psi} \gamma^{5} \gamma^{a} \psi$, two "charged currents", $\Lambda_{(+)}^{a}=\left(\psi_{c}\right)^{+} \alpha^{a} \psi=\Theta^{a}+i \Phi^{a}$ and $\Lambda_{(-)}^{a}=(\psi)^{+} \alpha^{a} \psi_{c}=\Theta^{a}-i \Phi^{a}$, the "scalar" $\mathcal{S}=\psi^{+} \rho_{1} \psi \equiv \bar{\psi} \psi$ and " pseudoscalar" $\mathcal{P}=\psi^{+} \rho_{2} \psi \equiv-i \bar{\psi} \gamma^{5} \psi$. Well-known are the six components of the skew-symmetric "tensor" $\Sigma^{a b}=(1 / 2) \psi^{+}\left[\alpha^{a} \rho_{1} \alpha^{b}-\alpha^{b} \rho_{1} \alpha^{a}\right] \psi$ (or its dual, $\left.\Sigma_{a b}^{\star}=(1 / 2) \epsilon_{a b c d} \Sigma^{c d}\right)$. All of them are interconnected by the so-called Fierz relations [10]. The charge-conjugated spinor is defined as $\psi_{c}=\boldsymbol{C} \psi^{*}$ with a real-valued matrix $\boldsymbol{C}$ (e.g., $\boldsymbol{C}=\rho_{2} \sigma^{2}$ ).

${ }^{2}$ This is a small subset of the Fierz identities that includes 28 basic relations and hundreds of derivable from them. They were studied in details in Ref. [10] as the basis for the mathematical reconstruction theorem [11] that states that Dirac spinor field can be uniquely restored via the Dirac currents (without any account for the dynamics). Within this approach it is possible to replace tetrad vectors of any coordinate system by an equivalent Dirac field thus simplifying various calculations [12]. Among the objects connected via the Fierz identities is present the skew-symmetric $\Sigma^{a b}$. The $\Sigma^{a b}$ appears to be a combination of the skew-symmetric products $j^{[a} \mathcal{J}^{b]}$ and $\Theta^{[a} \Phi^{b]}$ and scalars. The author was not aware of this fact and wrongfully tried [7] to employ $\Sigma^{a b}$ to build a substitute for the $\Theta^{a}$ and $\Phi^{a}$.

${ }^{3}$ Indeed, the necessary and sufficient condition for the linear independence is that the system of linear equations, $\sum_{A} c^{A} J_{A}=0$, has only a trivial solution, $c^{A}=0$; the latter is possible if and only if matrix that has these quadruples as its columns has a nonzero determinant, $\operatorname{det}\left|J_{A}^{a}\right| \neq 0$. The determinant of the $4 \times 4$ matrix $\left|J_{A}^{a}\right|$ equals $\operatorname{det}\left|J_{A}^{a}\right|=\mathcal{R}^{4}$, where $\mathcal{R}^{2}$ is the squared module of the complex number, $\mathcal{R}^{2}=|\mathcal{S}+i \mathcal{P}|^{2}$. When $\mathcal{R}^{2}>0$ the four vectors $\boldsymbol{J}_{A}(\mathrm{P})$ are linearly independent and can serve as a basis of vector space over $\mathbb{M}$. The condition $\mathcal{R}^{2}=0$ is equivalent to two real equations, $\mathcal{S}=\mathcal{P}=0$, which determine a singular two-dimensional surface in $\mathbb{R}^{4}$ (and thus on $\mathbb{M}$ ). 
and also that the $V_{A b}=\eta_{A B} V_{b}^{B}$ is the solution of the linear system, $V_{a}^{A} V_{A b}=\eta_{a b}$. Therefore, all indices are moved up and down by the Minkowski $\eta_{a b}$ or $\eta_{A B}$, which is nothing but a consequence of the Fierz identities. At every point $\mathrm{P} \in \mathbb{M}$, any quadruple of scalar fields $U^{a}(\mathrm{P})$, regardless of its origin, can be presented as a linear combination of the basic quadruples $V_{A}^{a}(\mathrm{P})$ determined by the Dirac field $\psi(\mathrm{P})$,

$$
U^{a}(\mathrm{P})=u^{A}(\mathrm{P}) V_{A}^{a}(\mathrm{P}), u^{A}(\mathrm{P})=U^{a}(\mathrm{P}) V_{a}^{A}(\mathrm{P}),
$$

where $u^{A}$ are the components of the $U^{a}$ with respect to the basis $V_{A}^{a}$.

4. An intermediate tetrad basis. The components $V_{A}^{a}(\mathrm{P})$ of a quadruple $V_{A}(\mathrm{P})$ clearly cannot be associated with a tangent vector like (2.1) simply because the former are defined only in terms of the invariant components straight in the principal manifold $\mathbb{M}^{4}$ (!), while definition of the latter requires a reference to an arithmetic $\mathbb{R}^{4}$, and its components are not invariant. Despite being complete, the system $V_{A}^{a}$ cannot immediately serve as a basis for the tangent vectors (2.1). Its completeness is purely algebraic by nature, while linear independence and completeness of the system $\boldsymbol{h}_{a}=h_{a}^{\mu} \partial_{\mu}$ is analytic and is always traced back to linear independence of the vectors of the basis $\left(\partial / \partial x^{\mu}\right)$ (the linear vector space over $\mathbb{R}^{1}: x^{\mu}=x^{\mu}(\lambda), \lambda \in \mathbb{R}^{1}$ ).

An invariant representation of vector $\boldsymbol{s}$ is possible only together with a system of the basic vectors $\boldsymbol{h}_{a}$; then it can be replaced by scalars, the tetrad components of the vector $\boldsymbol{s}, \boldsymbol{s}=\boldsymbol{h}_{a} s^{a}, s^{a}=h_{\mu}^{a} s^{\mu}$. Now, one can use (2.7) to expand the four scalars $s^{a}$ over the system $V_{A}^{a}$

$$
\boldsymbol{s}=\boldsymbol{h}_{a} V_{A}^{a} s^{A}=\boldsymbol{e}_{A} s^{A}, s^{A}=V_{a}^{A} h_{\mu}^{a} s^{\mu}
$$

and interpret the quantities $e_{A}^{\mu}=h_{a}^{\mu} V_{A}^{a}$ as the components of such a vector $\boldsymbol{e}_{A}=e_{A}^{\mu} \partial_{\mu}$ in coordinate basis that the scalars $V_{A}^{a}=e_{A}^{\mu} h_{\mu}^{a}$ are the components of $\boldsymbol{e}_{A}$ in the basis $\boldsymbol{h}_{a}$. The system of ODEs for the unknown $x^{\mu}$, $\mathrm{d} x^{\mu} / \mathrm{d} s^{A}=e_{A}^{\mu}$, defines the integral lines of the vector fields $\boldsymbol{e}_{A}$. It is also clear that the matrix $e_{\mu}^{A}=V_{a}^{A} h_{\mu}^{a}$ is the inverse of matrix $e_{A}^{\mu}$, viz. $e_{A}^{\mu} e_{v}^{A}=\delta_{v}^{\mu}$, and $e_{A}^{\mu} e_{\mu}^{B}=\delta_{A}^{B}$.

Let $\boldsymbol{s}$ in Equation (2.8) be one of the vectors of the basis $\boldsymbol{h}_{a}$ (or of the basis $\boldsymbol{e}_{A}$ ). Then $h_{b}^{\mu}=h_{a}^{\mu} V_{A}^{a} h_{b}^{A}$ and $e_{B}^{\mu}=e_{A}^{\mu} h_{a}^{A} V_{B}^{a}$, which results in

$$
h_{b}^{A} V_{A}^{a}=\delta_{b}^{a}, V_{B}^{a} h_{a}^{A}=\delta_{B}^{A} .
$$

Since $\operatorname{det}\left|V_{A}^{a}\right|=1$, the inverse matrix $V_{a}^{A}$ is uniquely defined; therefore,

$$
h_{a}^{A}(\mathrm{P})=V_{a}^{A}(\mathrm{P}), h_{A}^{a}(\mathrm{P})=V_{A}^{a}(\mathrm{P}) .
$$

The components of the tetrad vectors $\boldsymbol{h}_{a}(\mathrm{P})$ with respect to the basis $\boldsymbol{e}_{A}(\mathrm{P})$ must have invariant values (2.10). These equations together with normalization conditions (2.5) and unitarity, $\operatorname{det}\left|V_{A}^{a}\right|=1$, allow one to interpret $V_{A}^{a}(\mathrm{P})$ as the matrix of a local Lorentz rotation between the bases $\boldsymbol{e}_{A}(\mathrm{P})$ and $\boldsymbol{h}_{a}(\mathrm{P})$ with parameters that are determined by the Dirac field $\psi(\mathrm{P})^{4}$. So far, as long as we are confined to a point, we must refrain from associating this rotation with the physical Lorentz transformations of special relativity.

Since $V_{A}^{a}(\psi)$ are immediately defined as the fields over entire manifold $\mathbb{M}$, we expect that if two systems, $\boldsymbol{e}_{A}(\mathrm{P})$ and $\boldsymbol{h}_{a}(\mathrm{P})$, do exist, they are isomorphic not only in tangent $T_{p}$ but even as fields over $\mathbb{M}$. The question is whether the integral lines of the vector fields $\boldsymbol{h}_{a}(\mathrm{P})$ and/or $\boldsymbol{e}_{A}(\mathrm{P})$ can form a coordinate net.

5. An auxiliary fundamental tensor (not a metric). It takes simple algebra to verify that at the point $\mathrm{P} \in \mathbb{M}$ the objects

$$
g_{\mu v}=\eta_{a b} h_{\mu}^{a} h_{v}^{b}=\eta_{A B} e_{\mu}^{A} e_{v}^{B}, \quad g^{\mu \nu}=\eta^{a b} h_{a}^{\mu} h_{b}^{v}=\eta^{A B} e_{A}^{\mu} e_{B}^{v},
$$

can be used to move the coordinate (Greek) indices up and down. Indeed, $g_{\mu \nu} h_{b}^{v}=\eta_{c a} h_{\mu}^{c} h_{v}^{a} h_{b}^{v}=\eta_{c b} h_{\mu}^{c}=h_{b \mu}$.

\footnotetext{
${ }^{4}$ Long ago, E. Cartan [13] pointed to a difficulty, i.e. there are no representations of the general linear group of transformations $G L(4)$ that are similar to spinor representations of the Lorentz group of rotations. From the physical standpoint this argument is marginal since Lorentz transformations are between the reference frames of inertial observers and not between different differentiable mappings $\mathbb{M} \rightarrow \mathbb{R}^{4}$. Cartan stated the following theorem, which vetoed spinors in Riemannian geometry:

"With the geometric sense given to the word 'spinor' it is impossible to introduce spinors into classical Riemannian technique; i.e., having chosen an arbitrary system of co-ordinates $x^{\mu}$ for space, it is impossible to represent spinor by any finite number of components $\psi_{i}$ such that $\psi_{i}$ have covariant derivatives of the form $\psi_{i ; \mu}=\partial_{\mu} \psi_{i}+\Gamma_{i \mu}^{j} \psi_{j}$, where $\Gamma_{i \mu}^{j}$ are determinate functions of $x^{\mu}$." Of these two underscored reservations of Cartan, the first one was investigated by Ne'eman et al. [14], who proposed to overcome the veto by resorting to the infinite-dimensional representations of the Lorentz group. The present study explores the window, which is left open by the second reservation.
} 
With $g_{\mu v}$ thus defined, we also have the formal relations

$$
g_{\mu \nu} h_{a}^{\mu} h_{b}^{v}=\eta_{a b}, g_{\mu \nu} e_{A}^{\mu} e_{B}^{v}=\eta_{A B},
$$

which can be interpreted as orthonormality relations for the tetrad bases $h_{a}^{\mu}$ and $e_{A}^{\mu}$ if we postulate that this $g_{\mu v}$ determines a metric in coordinate basis. Indeed, by virtue of the identities (2.11) the equation,

$$
\mathrm{d} s^{2}=g_{\mu \nu} \mathrm{d} x^{\mu} \mathrm{d} x^{\nu}=\eta_{a b} \mathrm{~d} \lambda^{a} \mathrm{~d} \lambda^{b}=\eta_{A B} \mathrm{~d} S^{A} \mathrm{~d} S^{B},
$$

determines an interval which is Euclidean locally and invariant with respect to the choice of the coordinate basis within a domain where $\mathcal{R} \neq 0$. Most likely, this is not the metric that governs propagation of signals at a larger scale. It is remarkable that Fierz identities determine a system of unit vectors even before a notion of length is introduced.

Finally, when $g_{\mu v}$ is defined according to (2.10) and $R^{2}=0$ then all four vectors $\boldsymbol{e}_{\mathrm{A}}=V_{A}^{a} \boldsymbol{h}_{a}$, regardless of the tetrad $\boldsymbol{h}_{a}(x)$, which obviously does not have this property, also become lightlike on a two-dimensional surface, $\mathcal{S}=\mathcal{P}=0$, in spacetime $\mathbb{M}^{4}$. Obviously, in this case matrix $V_{A}^{a}$ has no inverse.

\section{Vector and Dirac Fields in Spacetime. Analytic Preliminaries}

From now on, we look at the $\psi_{\sigma}(\mathrm{P})$ as the physical Dirac field over four-dimensional manifold $\mathbb{M}$. The points $\mathrm{P} \in \mathbb{M}$ are mapped onto points $\left(x^{0}, x^{1}, x^{2}, x^{3}\right) \in \mathbb{R}^{4}$. The components $\psi_{\sigma}$ are thought of as smooth functions of the arbitrarily parameterized points $x^{\mu}=\left(x^{0}, x^{1}, x^{2}, x^{3}\right)$ of the spacetime. So far, we have verified that the algebraic structure of bilinear forms of the Dirac field naturally contains an orthogonal quadruple of unit (with respect to Minkowski metric) vectors at a generic point. By the argument of algebraic completeness, this quadruple must be isomorphic to a basis of any four non-complanar tangent vectors $\boldsymbol{h}_{a}(\mathrm{P})$ in $\mathbb{M}$. In a coordinate space $\mathbb{R}^{4}$, the latter are transformed as $d \mathbf{x}$, while the former are scalars. In $\mathbb{R}^{a}$, for a given fixed $\lambda$, we can consider $x^{\lambda}=$ const as the equation of a coordinate hypersurface and the lines along which all coordinates, but $x^{\lambda}$, are constant as coordinate lines. Tangent vectors of these lines (which are gradients of the linear function $\varphi(x)=x^{\lambda}$ ) are $h_{(\lambda)}^{\mu}=\partial x^{\mu} / \partial x^{\lambda}=\delta_{(\lambda)}^{\mu}$. Their covariant counterparts, $h_{\mu}^{(\lambda)}=\partial x^{\lambda} / \partial x^{\mu}=\delta_{\mu}^{(\lambda)}$, are the gradient vectors and the system of equations $\partial_{\mu} x^{(\lambda)}=h_{\mu}^{(\lambda)}(x)$ is integrable, but there is no metric and no way to determine if its coordinate lines are orthogonal. One may replace $x^{\mu}$ by smooth functions of other coordinates $y^{\mu}, x^{\mu}=f^{\mu}(y)$, thus redefining coordinate lines and surfaces, but such a change does not alter $\psi(x(y))$ and has nothing to do with "Lorentz rotations".

Thus, we have to account for two different kinds of invariance. One of them is the covariance, a trivial mathematical independence from the coordinate system. The second one is the invariance of the Dirac field as the matter, and it is dominant on every account, because any conceivable measurement requires the presence of the localized physical objects. In this section, we consider the Dirac field as a known function of coordinates and do not employ its equation of motion.

\subsection{Dirac Currents as a "Moving Frame" in Spacetime}

The Dirac field $\psi(\mathrm{P})$ is a coordinate scalar, but it naturally generates an affine centered vector space (spanned by the Dirac currents $\left.\boldsymbol{e}_{A}(\mathrm{P})\right)$ at $\mathrm{P}$, which is similar to the tangent space $T_{p}$ of the four-dimensional manifold $\mathbb{M}$ at $\mathrm{P}$ (spanned by the vectors $\left.\partial_{\mu}\right|_{\mathrm{P}}$ or $\boldsymbol{h}_{a}(\mathrm{P})$ ). These currents constitute a complete basis, they are of unit length and orthogonal in the sense of Equation (2.5). The continuous field of tetrad $\boldsymbol{e}_{A}(\mathrm{P})$ is embedded into $\mathbb{M}$. Therefore, an infinitesimal change of the $V_{A}^{a}$ (and, eventually, of the $\boldsymbol{e}_{A}$ ) from point $\mathrm{P}$ to point $\mathrm{P}^{\prime}$ is predetermined as,

$$
\mathrm{d} V_{A}^{a}\left(\mathrm{PP}^{\prime}\right)=\varpi_{A}^{B}\left(\mathrm{PP}^{\prime}\right) V_{B}^{a}(\mathrm{P}) .
$$

Also predetermined is the derivative of the scalars $V_{A}^{a}, \partial_{\mu} V_{A}^{a}(\mathrm{P})=\omega_{A \mu}^{B}(\mathrm{P}) V_{B}^{a}(\mathrm{P})$, and it has a very simple meaning. For a given displacement $\mathrm{d} x^{\mu}$ in $\mathbb{R}^{4}$, the total change $\mathrm{d} V_{A}^{a}=\partial_{\mu} V_{A}^{a}(\mathrm{P}) \mathrm{d} x^{\mu}$ can be expanded over a complete system $V_{B}(\mathrm{P})$ with the coefficients $\varpi_{A}^{B}\left(\mathrm{PP}^{\prime}\right)=\omega_{A \mu}^{B}(\mathrm{P}) \mathrm{d} x^{\mu}$. More precise is the directional derivative,

$$
\nabla_{\boldsymbol{h}} V_{A}^{a}(\mathrm{P})=\omega_{A \boldsymbol{h}}^{B}(\mathrm{P}) V_{B}^{a}(\mathrm{P}),
$$


along an arbitrary vector $\boldsymbol{h}$ in $\mathbb{M}$. By taking $\boldsymbol{h}=\boldsymbol{e}_{D}$, we immediately recognize the connections $\omega_{A D}^{B}(P)$, with the directional derivative, $\partial_{D}=e_{D}^{\mu} \partial_{\mu}$, along $\boldsymbol{e}_{D} \in \mathbb{M}$, as objects in principal manifold $\mathbb{M}$,

$$
\nabla_{D} V_{A}^{a}(\mathrm{P})=\omega_{A D}^{B}(\mathrm{P}) V_{B}^{a}(\mathrm{P}), \nabla_{D} V_{a}^{A}(\mathrm{P})=\omega_{B D}^{A}(\mathrm{P}) V_{a}^{B}(\mathrm{P}) .
$$

Then $\omega_{B D}^{A}=V_{a}^{A}\left(\nabla_{D} V_{B}^{a}\right)=-V_{B}^{a}\left(\nabla_{D} V_{a}^{A}\right)$. Since $\mathrm{d}\left(V_{a}^{A} V_{B}^{a}\right)=0\left[V_{a}^{A} V_{B}^{a}=\delta_{B}^{A}\right]$ we immediately conclude that

$$
\eta_{B C} \omega_{A D}^{C}=\omega_{A B D}=-\omega_{B A D},
$$

viz., the $\omega_{A B D} \in \mathbb{M}$ is skew-symmetric in the first two indices.

\subsection{Covariant Derivatives at a Point in $\mathbb{M}$}

In what follows, we compute the covariant derivatives of the vector and spinor components with respect to different bases and establish their interrelation.

1. The Dirac tetrad. Starting from Equations (2.7) and (3.3) and following the Cartan's idea of a moving frame [15], we can compute the covariant derivative of the components of any vector $\boldsymbol{u}(\mathrm{P})=\boldsymbol{u}^{A}(\mathrm{P}) \boldsymbol{V}_{A}(\mathrm{P})$,

$$
\partial_{D} \boldsymbol{u}(\mathrm{P})=\boldsymbol{V}_{A}(\mathrm{P})\left[\partial_{D} u^{A}(\mathrm{P})+\omega_{B D}^{A}(\mathrm{P}) u^{B}(\mathrm{P})\right],
$$

or, in terms of components with respect to the basis $\boldsymbol{e}_{A}$,

$$
\nabla_{D} u^{A}=\partial_{D} u^{A}+\omega_{B D}^{A} u^{B},
$$

where $\mathrm{d} u^{A}=\partial_{D} u^{A} \mathrm{~d} S^{D}$ are the relative changes of the components and $\nabla_{D} u^{A} \mathrm{~d} S^{D}$ is their total change. We explicitly see that the presence of the physical Dirac field over the principal manifold $\mathbb{M}$ immediately endows $\mathbb{M}$ with an affine connection. It also provides a natural definition of parallel transport as a transformation that leaves the components $u^{A}$ of a vector unchanged with respect to a local basis, even when the local tetrad (or a coordinate hedgehog) changes its orientation from point to point. Equation (3.3) is a special case of Equation (3.6) when $u^{A}=V_{a}^{A}$. Taking for $u^{A}$ the components of the vector current, $j^{A}=V_{a}^{A} j^{a}=\psi^{+} \alpha^{A} \psi$, one can define the covariant derivative of the Dirac field without leaving the principal manifold $\mathbb{M}$. Indeed, assuming that

$$
D_{A} \psi=\partial_{A} \psi-\Gamma_{A} \psi,
$$

and comparing with Equation (3.6) one readily obtains the equation that determines the connection $\Gamma_{A}[16]$,

$$
\Gamma_{D}^{+} \alpha^{A}+\alpha^{A} \Gamma_{D}=-\omega_{B D}^{A} \alpha^{B}=V_{B}^{a}\left(\nabla_{D} V_{a}^{A}\right) \alpha^{B},
$$

where $\alpha^{A}=V_{a}^{A} \alpha^{a}$ and these matrices $\alpha^{A}$, depending on $\psi$, must be considered as primary objects in $\mathbb{M}$.

2. Arbitrary tetrads. Knowing the affine connection in the basis of vectors $\boldsymbol{V}_{A}$, we can find it in any other basis $\boldsymbol{h}_{a}(P)$. Indeed, starting from Equation (3.6) we rewrite covariant derivative in terms of the basis vectors $\boldsymbol{h}_{a}$,

$$
V_{A}^{a} V_{d}^{D}\left(\nabla_{D} u^{A}\right)=\partial_{d} u^{a}+\gamma_{b d}^{a} u^{b} \equiv \nabla_{d} u^{a}(\mathrm{P}),
$$

where $\partial_{d}=h_{d}^{\mu} \partial_{\mu}$ and $\gamma_{b d}^{a}$ stands for the expression, $\gamma_{b d}^{a}=V_{B}^{a} \partial_{d} V_{b}^{B}+\omega_{B D}^{A} V_{A}^{a} V_{b}^{B} V_{d}^{D}$. By virtue of Equations (2.9), we have $\gamma_{b d}^{a}=V_{d}^{D}\left[\nabla_{D} V_{b}^{B}\right] V_{B}^{a}=h_{d}^{D}\left[\nabla_{D} h_{b}^{B}\right] h_{B}^{a}$. Using Equation (3.3), we obtain (by definition, $\partial_{D} V_{B}^{b}=$ $0 ; V_{B}^{b}$ is a matrix of Lorentz rotation),

$$
\gamma_{b d}^{a}=\omega_{B D}^{A} V_{A}^{a} V_{b}^{B} V_{d}^{D} .
$$

These invariants are nothing but the coefficients of rotation of the basic vectors $\boldsymbol{h}_{a}$ with respect to the basis $\boldsymbol{e}_{\mathrm{A}}$. Conversely, the equation,

$$
\omega_{B D}^{A}=-h_{D}^{d}\left[\partial_{d} h_{b}^{A}-\gamma_{b d}^{a} h_{a}^{A}\right] h_{B}^{b} \equiv h_{D}^{d}\left(\nabla_{d} h_{B}^{b}\right) h_{b}^{A}=V_{D}^{d}\left(\nabla_{d} V_{B}^{b}\right) V_{b}^{A},
$$

gives the coefficients of rotation $\omega_{B D}^{A}$ of the basic vectors $\boldsymbol{V}_{A}$ with respect to the basis $\boldsymbol{h}_{a}$.

3. Coordinate basis. In the coordinate picture, the basis vectors $\boldsymbol{h}_{a}(x)$ are assumed to be known in advance. 
In this case, one can derive the covariant derivative as

$$
h_{\mu}^{d} h_{a}^{v}\left(\nabla_{d} u^{a}\right)=\partial_{\mu} u^{v}+\Gamma_{\sigma \mu}^{v} u^{\sigma} \equiv \nabla_{\mu} u^{v},
$$

where $\Gamma_{\sigma \mu}^{v}$ stands for

$$
\Gamma_{\sigma \mu}^{v}=h_{b}^{v} \partial_{\mu} h_{\sigma}^{b}+\gamma_{b d}^{a} h_{\mu}^{d} h_{a}^{v} h_{\sigma}^{b} \equiv h_{\mu}^{d}\left(\nabla_{d} h_{\sigma}^{b}\right) h_{b}^{v} .
$$

and (because of the term with $\partial_{\mu} h_{\sigma}^{b}$ ) it is transformed as a connection under a change of the coordinates. Alternatively, we could start with $\nabla_{\mu}^{\mu} u^{V}=e_{\mu}^{D} e_{A}^{v}\left(\nabla_{D} u^{A}\right)$ (or just substitute $\gamma_{b d}^{a}$ from Equation (3.10)) and obtain another representation of the same connection $\Gamma_{\sigma \mu}^{v}$,

$$
\Gamma_{\sigma \mu}^{v}=e_{B}^{v} \partial_{\mu} e_{\sigma}^{B}+\omega_{B D}^{A} e_{\mu}^{D} e_{A}^{v} e_{\sigma}^{B} \equiv e_{\mu}^{D}\left(\nabla_{D} e_{\sigma}^{B}\right) e_{B}^{v},
$$

which is now expressed via quantities that explicitly depend on the physical Dirac field. Finally, using Equations (12), we can invert the last two equations to obtain,

$$
\gamma_{b d}^{a}=h_{d}^{\mu}\left(\nabla_{\mu} h_{b}^{v}\right) h_{v}^{a}, \omega_{B D}^{A}=e_{D}^{\mu}\left(\nabla_{\mu} e_{B}^{v}\right) e_{v}^{A},
$$

which is normally taken as an ad hoc definition of the coefficients of rotation of tetrad vectors when one prefers to stay in $\mathbb{R}^{4}$. Notably, Equations (3.15) and (3.3) determine the same $\omega_{B D}^{A}$, although Equation (3.3) apparently belongs to $\mathbb{M}^{4}$ and has nothing to do with the $\mathbb{R}^{4}$. This may be considered as an evidence that the vectors $h_{a}^{\mu}$ and the connections $\gamma_{b d}^{a}$ are the auxiliary quantities.

When $h^{\mu}$ is a vector and $g_{v \mu}(x)$ is a tensor (not necessarily determining a metric) then the covariant derivative $\nabla_{v} h^{\mu}$ with respect to $g_{v \mu}$ is also a tensor [17]. Using Equations (3.12) and (3.15), it is straightforward to check that if $g_{v \mu}(x)$ has the form (2.10) then $\nabla_{\lambda} g_{v \mu}=0$. Indeed, since $\gamma_{b a d}=-\gamma_{a b d}$ we have

$$
\nabla_{\lambda} g_{v \mu}=\eta^{a b}\left[h_{a \mu} \nabla_{\lambda} h_{b v}+h_{b v} \nabla_{\lambda} h_{a \mu}\right]=h_{\mu}^{a} h_{\nu}^{b} h_{\lambda}^{d}\left(\gamma_{b a d}+\gamma_{a b d}\right)=0 \text {. }
$$

An idea of how to find this $g_{v \mu}$ practically, will become clear only in the next paper [8], where a concrete solution $\psi$ is found. Starting from there, one can take the following path, $\psi \rightarrow V_{A}^{a} \rightarrow \omega_{A B C} \rightarrow \gamma_{a b c} \rightarrow h_{a}^{\mu} \rightarrow e_{A}^{\mu}$ and, eventually, explicitly determine the $g_{\mu v}(\psi(x))$.

4. Connections for the Dirac field. Starting from Equation (3.9) for the vector current $j^{a}=\psi^{+} \alpha^{a} \psi$,

$$
D_{b} j^{a}=\partial_{b} j^{a}+\gamma_{c b}^{a} j^{c} \equiv \nabla_{b} j^{a}, D_{b} j_{a}=\partial_{b} j_{a}-\gamma_{a b}^{c} j_{c} \equiv \nabla_{b} j_{a} .
$$

or translating Equation (3.8) into the basis $\boldsymbol{h}_{a}$, it is straightforward to obtain the following equation for the matrix $\Gamma_{a}{ }^{5}$ :

$$
\Gamma_{d}^{+} \alpha_{a}+\alpha_{a} \Gamma_{d}=\gamma_{a d}^{b} \alpha_{b}, \Gamma_{d}^{+} \alpha^{a}+\alpha^{a} \Gamma_{d}=-\gamma_{b d}^{a} \alpha^{b},
$$

where $\Gamma_{d}=V_{d}^{D} \Gamma_{D}$, and nothing implies that $\alpha^{a}$ must be numerical matrices ${ }^{6}$. If we introduce $\alpha^{v}=h_{a}^{v} \alpha^{a}=$ $e_{A}^{v} \alpha^{A}$ and $\Gamma_{\mu}=h_{\mu}^{d} \Gamma_{d}=e_{\mu}^{D} \Gamma_{D}$ and use (3.15), then Equations (3.8) and (3.17) can be rewritten entirely in $\mathbb{R}^{4}$,

$$
\Gamma_{\mu}^{+} \alpha^{v}+\alpha^{v} \Gamma_{\mu}=-\nabla_{\mu} \alpha^{v},
$$

Equations (3.17) and (3.18) indicate that the Dirac matrices $\alpha^{a}$ are covariantly constant with respect to the "connection" $\Gamma_{a}$ of the Dirac field, $D_{b} \alpha^{a}=D_{\mu} \alpha^{\mu}=0$. The same is true for other representations as well.

Either of Equations (3.8), (3.17) and (3.18) can be solved (algebraically) for the corresponding $\Gamma_{\kappa}$. The most general solution reads as

$$
\Gamma_{d}=i e A_{d}+i g \rho_{3} \mathcal{W}_{d}+(1 / 4) \gamma_{a b d} \rho_{1} \alpha^{a} \rho_{1} \alpha^{b},
$$

where, so far, $e$ and $g$ are arbitrary constants. The term $i e A_{d}$ in the connection (19) (or the field $A_{\mu}$ ) is ${ }^{5}$ Indeed, multiplying both sides by $V_{d}^{D} V_{A}^{a}$ we will have in the r.h.s. $V_{d}^{D} V_{A}^{a} V_{B}^{h}\left(\nabla_{D} V_{h}^{A}\right) \alpha^{B}=-V_{d}^{D} V_{A}^{a} V_{h}^{A}\left(\nabla_{D} V_{B}^{h}\right) \alpha^{B}=-V_{d}^{D}\left(\nabla_{D} V_{B}^{a}\right) V_{b}^{B} \alpha^{b}$ $=\gamma_{b d}^{a} \alpha^{b}$.

${ }^{6}$ This is straightforward to show, $\begin{aligned} \partial_{b}\left(\psi^{+} \alpha^{a} \psi\right)+\gamma_{c b}^{a}\left(\psi^{+} \alpha^{a} \psi\right) & \equiv \nabla_{b}\left(\psi^{+} \alpha^{a} \psi\right)=D_{b}\left(\psi^{+} \alpha^{a} \psi\right)=\left(D_{b} \psi\right)^{+} \alpha^{a} \psi+\psi^{+}\left(D_{b} \alpha^{a}\right) \psi+\psi^{+} \alpha^{a} D_{b} \psi \\ & =\partial_{b}\left(\psi^{+} \alpha^{a} \psi\right)-\psi^{+}\left(\Gamma_{b}^{+} \alpha^{a}+\alpha^{a} \Gamma_{b}\right) \psi+\psi^{+}\left[\Gamma_{b}^{+} \alpha^{a}+\alpha^{a} \Gamma_{b}+\gamma_{c b}^{a} \alpha^{c}\right] \psi\end{aligned}$
where $D_{b} \alpha^{a}=\partial_{b} \alpha^{a}+\Gamma_{b}^{+} \alpha^{a}+\alpha^{a} \Gamma_{b}+\gamma_{c b}^{a} \alpha^{c}=\nabla_{b} \alpha^{a}+\Gamma_{b}^{+} \alpha^{a}+\alpha^{a} \Gamma_{b}$. 
unquestionably interpreted as the electromagnetic potential. The term $\operatorname{ig} \rho_{3} \mathcal{W}_{d}$ (or field $\mathcal{W}_{u}$ ) could have been interpreted as another field that interacts with the axial current $J^{\mu 7}$. The connection (3.19) commutes with the matrix $\rho_{3}$, so that Equation (3.17) remains the same when $\alpha_{a} \rightarrow \rho_{3} \alpha_{a}$. So far, it neither commutes nor anticommutes with $\rho_{1}$ and $\rho_{2}$, viz.

$$
\Gamma_{b}^{+} \rho_{1}+\rho_{1} \Gamma_{b}=2 g \rho_{2} \mathcal{W}_{b}, \quad \Gamma_{b}^{+} \rho_{2}+\rho_{2} \Gamma_{b}=-2 g \rho_{1} \mathcal{W}_{b} .
$$

Similar formulae arise for the charge-conjugated connection. Since $\boldsymbol{C} \rho_{1} \boldsymbol{C}^{-1}=-\rho_{1}$ and $\left[\boldsymbol{C} \alpha^{i} \boldsymbol{C}^{-1}\right]^{*}=\alpha^{i}$,

$$
\Gamma_{b}^{+} \alpha_{a}+\alpha_{a} \Gamma_{b}^{c}=\gamma_{a b}^{d} \alpha_{d}-2 i e A_{b} \alpha_{a},\left[\Gamma^{c}\right]_{b}^{+} \alpha_{a}+\alpha_{a} \Gamma_{b}=\gamma_{a b}^{d} \alpha_{d}+2 i e A_{b} \alpha_{a} .
$$

The commutation relations for the Dirac matrices $\alpha^{\mu}=h_{a}^{\mu} \alpha^{a}$ and $\alpha^{A}=V_{a}^{A} \alpha^{a}$ are

$$
\alpha^{\mu} \rho_{1} \alpha^{\nu}+\alpha^{v} \rho_{1} \alpha^{\mu}=2 \rho_{1} g^{\mu \nu} \text { and } \alpha^{A} \rho_{1} \alpha^{B}+\alpha^{B} \rho_{1} \alpha^{A}=2 \rho_{1} \eta^{A B}
$$

in $\mathbb{R}^{4}$ and $\mathbb{M}$, respectively. We assume that the matrices $\alpha^{a}$ are associated with the basis $\boldsymbol{h}_{a}$ in the tangent $T_{p}$, while matrices $\alpha^{A}$ belong to the principal manifold $\mathbb{M}$. In what follows, we consider Dirac field as the primary matter field; covariant derivatives of its bilinear functions will be computed only using Equations (3.17)-(3.19).

5. Connections in different bases. Equations (3.10) and (3.11) are nothing but the well known formulae for transformation of a linear connection between two non-coordinate (anholonomic) bases. In these bases, all quantities are functions of the point $\mathrm{P}$ in the principal manifold $\mathbb{M}$, and thus independent of the coordinate basis in the $\mathbb{R}^{4}$. For example, we readily have the coordinate-independent equation of the parallel transport of a vector $\boldsymbol{u}$ along a vector $\boldsymbol{v}=\mathrm{d} / \mathrm{d} \lambda$, viz. $\nabla_{v} \boldsymbol{u}(\mathrm{P})=v^{A} \nabla_{A} \boldsymbol{u}(\mathrm{P})=v^{a} \nabla_{a} \boldsymbol{u}(\mathrm{P})=0$.

If we omit indices and use the notation $\mathcal{U}$ for matrix $V_{A}^{a}$ (as well as $\mathcal{U}^{-1}$ for $V_{a}^{A}, \Gamma_{\kappa}$ for $\gamma_{b \kappa}^{a}$ and $\Omega_{\kappa}$ for $\omega_{B \kappa}^{A}$ ) then Equations (3.10) and (3.11) read as

$$
\Gamma_{\kappa}=\mathcal{U}^{-1} \Omega_{\kappa} \mathcal{U}-\mathcal{U}^{-1} \partial_{\kappa} \mathcal{U}, \Omega_{\kappa}=\mathcal{U} \Gamma_{\kappa} \mathcal{U}^{-1}-\mathcal{U} \partial_{\kappa} \mathcal{U}^{-1},
$$

which are the universal expressions ${ }^{8}$ for all kinds of connections associated with local transformations. Equations (3.6) and (3.9), augmented by definition of the derivatives, $\partial_{d}=h_{d}^{\mu} \partial_{\mu}$ and $\partial_{D}=V_{D}^{d} \partial_{d}=e_{D}^{\mu} \partial_{\mu}$, are fixing the components of any vector with respect to the (moving) tetrads $\boldsymbol{e}_{A}$ and $\boldsymbol{h}_{a}$. The existence of the field of unitary matrix of the Lorentz transform $\mathcal{U}=\left|V_{A}^{a}\right|$ (and then of an affine connection $\omega_{B D}^{A}$ ) appears to be an amazing consequence of the Fierz identities for bilinear forms of the Dirac field. Finally, it is straightforward to check that, once $h_{a}^{\mu}$ and $e_{A}^{\mu}$ are the components of vectors and $\gamma_{b a d}$ and $\omega_{B A D}$ are scalars, the connection $\Gamma_{\sigma \mu}^{v}$ transforms under a further change of the coordinates as

$$
\frac{\partial x^{v}}{\partial x^{\nu^{\prime}}} \Gamma_{\sigma^{\prime} \mu^{\prime}}^{v^{\prime}}=\frac{\partial x^{\sigma}}{\partial x^{\sigma^{\prime}}} \frac{\partial x^{\mu}}{\partial x^{\mu^{\prime}}} \Gamma_{\sigma \mu}^{v}+\frac{\partial^{2} x^{\nu}}{\partial x^{\sigma^{\prime}} \partial x^{\mu^{\mu^{\prime}}}},
$$

which guarantees that the derivative $\nabla_{\mu} u^{v}$ transforms as a tensor. Transformations (3.10) and (3.11) are reduced to this formula when the tetrads are formed by the gradient vectors.

By definition, $\gamma_{a b d}=\left(\nabla_{d} h_{a}^{\kappa}\right) h_{b \kappa}$, were index $\kappa$ can belong to any of the bases. Therefore, Equation (3.19) has the required general form (3.22) and can be rewritten as $\Gamma_{d}=i e A_{d}+i g \rho_{3} \mathcal{W}_{d}+(1 / 4) \nabla_{d}\left(\rho_{1} \alpha^{a} h_{a}^{\kappa}\right) \rho_{1} \alpha^{b} h_{b \kappa}$ in

\footnotetext{
${ }^{7}$ In the early days of the Dirac theory, it was firmly established that $\mathcal{S}=\psi^{+} \rho_{1} \psi$ and $\mathcal{P}=\psi^{+} \rho_{2} \psi$ are Lorentz scalars, which, however, does not guarantee that they are scalars with respect to the general coordinate transformations of the group GL(4). V. Fock [16] resorted to a specific choice of the Dirac matrices to demonstrate that $S^{+} \rho_{1} S=\rho_{1}$ and $S^{+} \rho_{2} S=\rho_{2}$ under special Lorentz transformations $\mathrm{S}$. For now, we shall refer to the differential identity (4.4), $\nabla_{\mu} \mathcal{J}^{\mu}=2 m \mathcal{P}$; since $\mathcal{J}^{\mu}$ is a vector and $\nabla_{\mu} \mathcal{J}^{\mu}$ is a coordinate scalar, so are $\mathcal{P}$ and then $\mathcal{S}$ (due to the Fierz identity (2.3)). This argument is not geometric in its nature, because it relies on the equation of motion. Intriguing is that $\mathcal{P}$ and $\mathcal{S}$ are the coordinate scalars only due to equations of motion. At the moment, we have no convincing argument that would allow one to reject the presence of $\mathcal{W}_{d}$ in the $\Gamma_{d}$ except that we have no experimental evidence that $\mathcal{W}$ exists as a physical field. Here, such an argument is reached later (with the reference to the equations of motion) from the physical (and then mathematical) requirement that nothing in physical manifold $\mathbb{M}$ or in coordinate space $\mathbb{R}^{4}$ can depend on a tetrad basis $\boldsymbol{h}_{a}$. For the sake of clarity, some equations will be ending with “ $\cdots=0,\left\{=\mathcal{W}_{a}\right\}$ ”, until we reach Equations (4.16) and (4.21) and then prove that $\mathcal{W}_{a}=0$.

${ }^{8}$ In general. $\kappa$ is not a tetrad index.
} 
tetrad basis and as $\Gamma_{\mu}=i e A_{\mu}+i g \rho_{3} \mathcal{W}_{\mu}+(1 / 4) \nabla_{\mu}\left(\rho_{1} \alpha^{v}\right) \rho_{1} \alpha_{v}$ in the coordinate $\mathbb{R}^{4}$.

6. Symmetry of the connection $\Gamma_{\sigma \mu}^{v}$. If we naively assume that the Minkowski signature $\eta_{a b}$ in Equations (2.4) and (2.5) determines the local metric of an inertial reference frame at point $\mathrm{P}$ (with local coordinates $y^{a}$ ) and that $g_{v \mu}(\mathrm{P})$ of Equations (2.10) is obtained by a local coordinate transformation of the $\eta_{a b}$ then, being a tensor, the skew-symmetric part $\Gamma_{[\mu v]}^{\sigma}$ of the connection (the tensor of torsion) should be zero. This argument would require, in its turn, that the covariant tetrad vectors be the gradient vectors, $h_{\mu}^{a}(\mathrm{P})=\partial y^{a} /\left.\partial x^{\mu}\right|_{\mathrm{P}}$, which is by no means self-evident.

There is, however, another reason for the symmetry of $\Gamma_{\mu \nu}^{\sigma}$, which is hinted by the Cartan's method of moving frame. The field of tetrad $\boldsymbol{e}_{A}(\mathrm{P})$ belongs to $\mathbb{M}$ and can be used as a "moving frame" for all vectors $\boldsymbol{s} \in \mathbb{M}$, including the vectors $\mathrm{d} \mathbf{x}$ of infinitesimal displacements. Consider now a closed path $\mathbb{R}^{1} \subset \mathbb{M}$ through the point $\mathrm{P}_{0}$ and attach the " natural" tetrad $\boldsymbol{e}_{A}(\mathrm{P})$ to its points. Then every next point of the path has a position with respect to the tetrad of the previous point. Since the tetrad $\boldsymbol{e}_{A}(\mathrm{P})$ is changing from point to point, we have no other choice but to specify the transport of a vector as the parallel Fermi transport (in the sense that the components of a vector with respect to the local tetrad do not change) along the chosen path. We will be able to get back to $\mathrm{P}_{0}$ (the image of the path in the moving frame will be closed) with the same $\psi\left(\mathrm{P}_{0}\right)$ and, therefore, with the same tetrad $\boldsymbol{e}_{A}(\mathrm{P})$ and matrix $V_{A}^{a}\left(\mathrm{P}_{0}\right)$, which is imperative, if and only if the components $\Gamma_{\mu \nu}^{\sigma}$ of the connection, as they are defined in the coordinate basis $\partial / \partial x^{\mu}$ of the $\mathbb{R}^{4}$, are symmetric in their subscripts. Then the torsion tensor vanishes, and only then will we be able to contract the entire path to the point $\mathrm{P}_{0} \in \mathbb{M}$. Consequently, the following formulae,

$$
\left(\partial_{a} \partial_{b}-\partial_{b} \partial_{a}\right) \psi=-\left(\gamma_{a b}^{d}-\gamma_{b a}^{d}\right) \partial_{d} \psi,\left(\partial_{A} \partial_{B}-\partial_{B} \partial_{A}\right) \psi=-\left(\omega_{A B}^{D}-\omega_{B A}^{D}\right) \partial_{D} \psi .
$$

can be confidently used for any coordinate scalar $\psi(x)$.

\section{Differential Identities for the Dirac Currents}

As it was pointed out above, Equations (3.6) and (3.9) with the predetermined coefficients of rotation fix the components of a vector with respect to an a priori arbitrary tetrad basis. One might expect that these equations can be trivially used to fix the components of any tensor field. However, the coefficients of rotation of the "geometric tetrad" $h_{a}^{\mu}$ and those of the tetrad $e_{a}^{\mu}$ of the normalized Dirac currents are interconnected by Equation (3.10). Hence, the dynamic can potentially limit a feasible choice of the basis $h_{a}^{\mu}$. The coordinate system (coordinate lines) can be not arbitrary; not all coordinate variables can even have the meaning of coordinates. Therefore, it seems appropriate to postpone, for as long as possible, explicit use of any coordinate basis and treat the tetrad $e_{(A)}^{\mu}[\psi(x)]$ as an orthogonal moving frame [15]. An affine geometry will be constructive if and only if all the coefficients $\omega_{A C}^{B}$ of rotation of the tetrad $\boldsymbol{e}_{A}$ can be determined from the equations of motion.

In this section we show that this is indeed possible. There appears to be sufficient number of identities for the Dirac currents to completely determine the coefficients $\omega_{A C}^{B}$ and the connections $\Gamma_{B}$ in the covariant derivative $D_{B} \psi=\left(\partial_{B}-\Gamma_{B}\right) \psi$. Therefore, from now on we are dealing with the physical material Dirac field that satisfies the Dirac equations of motion,

$$
\alpha^{a} D_{a} \psi=-i m \rho_{1} \psi, \quad \psi^{+} D_{a}^{+} \alpha^{a}=i m \psi^{+} \rho_{1},
$$

with the derivative $D_{a}=\partial_{a}-\Gamma_{a}$, connection $\Gamma_{a}$ defined by Equation (3.19), and the mass parameter $m$. The latter is, for now, real, arbitrary and stands for the rate of mixing between the right and left components of the Dirac spinor. The equations of motion for the charge-conjugated spinor are

$$
\alpha^{a} D_{a}^{c} \psi_{c}=-i m \rho_{1} \psi_{c},\left[\psi_{c}\right]^{+} \overleftarrow{D}_{a}^{c+} \alpha^{a}=i m\left[\psi_{c}\right]^{+} \rho_{1},
$$

where $D_{a}^{c} \psi^{c}=\left(\partial_{a}-\Gamma_{a}^{c}\right) \psi^{c}$ is the covariant derivatives of the charge-conjugate Dirac field, and $\Gamma_{a}^{c}$ is given by Equations (3.21).

\subsection{Divergences of the Dirac Currents}

From the equations of motion (4.1) one immediately derives two well-known identities. Multiplying the Dirac equation by $\psi^{+}$from the left and its conjugate by $\psi$ from the right and taking their sum we readily obtain that 


$$
D_{a} j^{a}=\nabla_{\mu} j^{\mu}=0 .
$$

This equation clearly indicates conservation of the timelike vector current (of probability) of the Dirac field. The second identity is obtained from the Dirac Equation (4.1), which is multiplied by $\rho_{3}$ from the left (and its conjugate from the right, and noting that $\left.\rho_{3} \rho_{1}=-\rho_{1} \rho_{3}=i \rho_{2}\right)$. It indicates that the spacelike axial current is not conserved,

$$
D_{a} \mathcal{J}^{a}=\nabla_{\mu} \mathcal{J}^{\mu}=2 m \mathcal{P}
$$

and has the pseudoscalar density as a source. Since $\mathcal{P}$ is localized not less than $\mathcal{R}$, and the vector $\mathcal{J}^{\mu}$ is spacelike, it defines the radial direction. The existence of such a direction is a distinct characteristic of any localized object.

Similar identities can be derived for the vectors $\Theta^{a}$ and $\Phi^{a}$ of Section 2. Using Equations (3.21) and (4.2), we immediately arrive to covariant derivatives of the matrix elements $\Lambda_{a}^{( \pm)}$as

$$
D_{b} \Lambda_{a}^{( \pm)}=\partial_{b} \Lambda_{a}^{( \pm)}-\gamma_{a b}^{d} \Lambda_{d}^{( \pm)} \mp 2 i e A_{b} \Lambda_{a}^{( \pm)} \equiv \nabla_{b} \Lambda_{a}^{( \pm)} \mp 2 i e A_{b} \Lambda_{a}^{( \pm)} .
$$

Though these vectors are complex and explicitly depend on the phase of $\psi$, this dependence is compensated in the covariant derivative (4.5) by the gauge transformation of the vector potential. The derivatives of $\Theta$ and $\Phi$ become

$$
D_{b} \Theta_{a}=\nabla_{b} \Theta_{a}+2 e A_{b} \Phi_{a}, D_{b} \Phi_{a}=\nabla_{b} \Phi_{a}-2 e A_{b} \Theta_{a} .
$$

The fields of complex currents $\Theta^{a} \pm i \Phi^{a}$ look like being "charged” with a charge $2 e$. From the equations of motion (4.2) and using Equation (4.6), it is straightforward to get $D_{a} \Lambda_{( \pm)}^{a}=0$ and, consequently,

$$
\nabla_{\mu} \Theta^{\mu}=-2 e A_{\mu} \Phi^{\mu}, \quad \nabla_{\mu} \Phi^{\mu}=2 e A_{\mu} \Theta^{\mu} .
$$

Similarly to the vector of axial current, these vectors are not conserved due to electromagnetic potential $A_{\mu}$.

\subsection{Curls of the Dirac Currents}

In order to access the differential identities for the curls of the Dirac currents one has to compute, using the equations of motion, the derivatives of the objects $T_{a}^{a}, P_{a}^{a}, \Theta_{a}^{a}$, $\Phi_{a}^{a}$, which are traces of tensors (objects), $T_{b}^{a}=\psi^{+} \alpha^{a} D_{b} \psi, P_{b}^{a}=\psi^{+} \rho_{3} \alpha^{a} D_{b} \psi, \Theta_{b}^{a}=\left(\psi^{c}\right)^{+} \alpha^{a} D_{b} \psi$ and $\Phi_{b}^{a}=\left(\psi^{c}\right)^{+} \overleftarrow{D}_{b}^{c+} \alpha^{a} \psi$, respectively. These tensors are neither real nor symmetric, and we are not concerned here about their physical interpretation.

1. $T_{v}^{\mu}$-a tensor or not? One would expect the absolute differential of $T_{b}^{a}$, being computed according to the Leibniz rule, be as follows,

$$
D_{c} T_{b}^{a}=\partial_{c} T_{b}^{a}+\gamma_{d c}^{a} T_{b}^{d}-\gamma_{b c}^{d} T_{d}^{a} \equiv \nabla_{c} T_{b}^{a} .
$$

and this expression would fix, similarly to Equations (3.9) and (3.12), the components of the tensor $T_{\mu}^{\sigma}=h_{a}^{\sigma} h_{\mu}^{b} T_{b}^{a}$ with respect to the tetrad $\boldsymbol{h}_{a}$. If this expectation turns out justified then the usual covariant derivative will be immediately reproduced as

$$
\partial_{\lambda} T_{\mu}^{\sigma}+\Gamma_{v \lambda}^{\sigma} T_{\mu}^{v}-\Gamma_{\mu \lambda}^{v} T_{v}^{\sigma}=e_{\lambda}^{c} e_{a}^{\sigma} e_{\mu}^{b} \nabla_{c} T_{b}^{a}=\nabla_{\lambda} T_{\mu}^{\sigma}
$$

Contrary to the expectation of (4.8), the answer reads

$$
\begin{aligned}
D_{c}\left[\psi^{+} \alpha^{a} \vec{D}_{b} \psi\right] & =\partial_{c}\left[\psi^{+} \alpha^{a} \vec{D}_{b} \psi\right]-\psi^{+}\left[\Gamma_{c}^{+} \alpha^{a}+\alpha^{a} \Gamma_{c}\right] \vec{D}_{b} \psi \\
& =\partial_{c}\left[\psi^{+} \alpha^{a} \vec{D}_{b} \psi\right]+\gamma_{d c}^{a} \psi^{+} \alpha^{d} \vec{D}_{b} \psi
\end{aligned}
$$

with the last term of Equation (4.8) missing, and no hope to recover the full geometric expression (4.9) of the covariant derivative of the tensor! Contracting here indices $a$ and $c$ and using equations of motion we would arrive at [7]

$$
\partial_{a} T_{b}^{a}+\gamma_{c a}^{a} T_{b}^{c}-\gamma_{b a}^{c} T_{c}^{a}=-\gamma_{a b}^{c} T_{c}^{a}-i \psi^{+} \alpha^{a} \mathbb{D}_{a b} \psi .
$$


with the normal covariant derivative in the l.h.s. The $\mathbb{D}_{a b}$ and an abnormal term $\gamma_{a b}^{c} T_{c}^{a}$ in the r.h.s. originate from the commutator of the covariant derivatives, $\left[\vec{D}_{a}, \vec{D}_{b}\right]$. Its real part is the Lorentz force,

$\operatorname{Re}\left[i \psi^{+} \alpha^{a} \mathbb{D}_{a b} \psi\right]=e j^{a} F_{a b} \quad[7][16]^{9}$.

2. Abnormal terms and how they restore the GL(4) covariance. The abnormal term enters another identity that follows from the Dirac equation, which arises after contracting indices $a$ and $b$ in Equation (4.10). On the one hand, we formally have (Cf. footnote ${ }^{7}$. The $S=\psi^{+} \rho_{1} \psi$ must be a scalar and the last term in the r.h.s. must be absent.)

$$
D_{c}\left[\psi^{+} \alpha^{a} \vec{D}_{a} \psi\right]=\partial_{c}\left[\psi^{+} \alpha^{a} \vec{D}_{a} \psi\right]+\gamma_{b c}^{a} \psi^{+} \alpha^{b} \vec{D}_{a} \psi=-i m \partial_{c}\left[\psi^{+} \rho_{1} \psi\right]+\gamma_{b c}^{a} \psi^{+} \alpha^{b} \vec{D}_{a} \psi
$$

On the other hand, by virtue of the Dirac equation, the first term on the r.h.s. of (4.12) becomes

$\partial_{c}\left[-i m \psi^{+} \rho_{1} \psi\right]$. Alternatively, one can immediately use the equations of motion on the l.h.s. and only then differentiate,

$$
D_{c}\left[\psi^{+} \alpha^{a} \vec{D}_{a} \psi\right]=-i m D_{c}\left[\psi^{+} \rho_{1} \psi\right]=-i m \partial_{c}\left[\psi^{+} \rho_{1} \psi\right]+i m \psi^{+}\left[\Gamma_{c}^{+} \rho_{1}+\rho_{1} \Gamma_{c}\right] \psi .
$$

Comparing the last two equations and using (3.20), we finally find that the abnormal term $\gamma_{a b}^{c} T_{c}^{a}=h_{b}^{\lambda}\left(\nabla_{\lambda} h_{(a)}^{v}\right) h_{\sigma}^{(a)} T_{v}^{\sigma}$ vanishes (or at least can be expressed via abnormal field $\mathcal{W}_{b}$ )

$$
-\gamma_{a b}^{c} \cdot T_{c}^{a}=0, \quad\left\{=2 m g \mathcal{P} \mathcal{W}_{a}\right\},
$$

thus restoring the covariance of Equation (4.11). Remarkably, the usual covariance in coordinate space is restored due to equations of motion. Equation (4.14) yields two nontrivial conditions on the structure of the Dirac currents as follows. The Ricci coefficients are real-valued and skew-symmetric in the first two indices. The r.h.s. of Equation (4.14) is real. Therefore, the imaginary part of Equation (4.14) reads as

$$
\gamma_{a c b} \operatorname{Im}\left(T_{a c}-T_{c a}\right)=\gamma_{a c b}\left[D_{c}\left(\psi^{+} \alpha_{a} \psi\right)-D_{a}\left(\psi^{+} \alpha_{c} \psi\right)\right]=\gamma_{a c b}\left(\nabla_{c} j_{a}-\nabla_{a} j_{c}\right)=0 .
$$

In order to facilitate further analysis of the real part of Equation (4.14), let us rewrite its l.h.s. in terms of the axial current. Using the dual representation of the axial current as $\epsilon^{\text {stua }} \mathcal{J}_{a}=i \psi^{+} \alpha^{s} \rho_{1} \alpha^{t} \rho_{1} \alpha^{u} \psi,(s, t, u, \neq)$ and employing the equations of motion we obtain,

$$
D_{u} \epsilon^{\text {stua }} \mathcal{J}_{a}=-i \psi^{+} \alpha^{s} \vec{D}_{t} \psi+i \psi^{+} \alpha^{t} \vec{D}_{s} \psi-i \psi^{+} \overleftarrow{D}_{s}^{+} \alpha^{t} \psi+i \psi^{+} \overleftarrow{D}_{t}^{+} \alpha^{s} \psi,
$$

where the r.h.s is four times the anti-symmetric Hermitian part of the energy momentum tensor. Therefore, the real part of Equation (4.14) reads as

$$
(1 / 4) \gamma_{a c b} \cdot \epsilon^{a c s t} \cdot \nabla_{s} \mathcal{J}_{t}=(1 / 2){ }^{\star} \gamma_{a c b} \nabla_{c} \mathcal{J}_{a}=0 \quad\left\{=2 m g \mathcal{P} \mathcal{W}_{b}\right\}
$$

3. More non-tensors and abnormal terms. Next, consider the stress tensor $P_{b}^{a}=i \psi^{+} \rho_{3} \alpha^{a} D_{b} \psi$, mostly following the same protocol and starting from its covariant derivative. We find that

$$
D_{c}\left[\psi^{+} \rho_{3} \alpha^{a} \vec{D}_{b} \psi\right]=\partial_{c}\left[\psi^{+} \alpha^{a} \vec{D}_{b} \psi\right]-\gamma_{d c}^{a} \psi^{+} \rho_{3} \alpha^{d} \vec{D}_{b} \psi .
$$

Once again, the last term of Equation (4.8) is missing, and thus we have no confidence that the covariant derivative is a tensor. For the immediate purpose of this work, we only need the equations that emerge after contracting indices $a$ and $b$ in Equation (4.17),

$$
D_{c}\left[\psi^{+} \rho_{3} \alpha^{a} \vec{D}_{a} \psi\right]=\partial_{c}\left[\psi^{+} \rho_{3} \alpha^{a} \vec{D}_{a} \psi\right]+\gamma_{b c}^{a} \psi^{+} \rho_{3} \alpha^{b} \vec{D}_{a} \psi .
$$

By virtue of the Dirac equations, the first term in the r.h.s. becomes $\partial_{c}\left[m \psi^{+} \rho_{2} \psi\right]$. Alternatively, one can immediately use the equations of motion in the l.h.s. and only then differentiate (matrices $\rho_{3}$ and $\alpha^{a}$ commute),

\footnotetext{
${ }^{9}$ Three remarks are to be made here: 1 ) the Lorentz force in the r.h.s. allows one to associate the observable $j$ and $\mathcal{R}$ with a variations of the charge density even without reference to the Maxwell equations. A uniform distribution is not distinct from vacuum; 2) if the basis $h_{a}^{\mu}$ were holonomic, viz. $\quad \gamma_{b a}^{c}-\gamma_{a b}^{c}=0$, then there would have been no way to achieve the desired covariance. In fact, the abnormal term will vanish, but only if the nontrivial conditions (14) are met; 3) in general, $\mathbb{D}_{a b}=-(1 / 4) R_{a b c d} \rho_{1} \alpha^{c} \rho_{1} \alpha^{d}+i e F_{a b}$, where $R_{a b c d}$ and $F_{a b}$ are the Riemannian curvature and the electromagnetic field tensors, respectively.
} 


$$
D_{c}\left[\psi^{+} \rho_{3} \alpha^{a} \vec{D}_{a} \psi\right]=m D_{c}\left[\psi^{+} \rho_{2} \psi\right]=m \partial_{c}\left[\psi^{+} \rho_{2} \psi\right]+m \cdot 2 g S \mathcal{S} \mathcal{W}_{c} .
$$

Comparing the last two equations we finally get the equation,

$$
-\gamma_{c b}^{a} \cdot P_{a}^{c}=-2 \text { igmSW }_{b},
$$

which is complementary to Equation (4.14). Since $\gamma_{a c b}$ is skew-symmetric in the first two indices, the imaginary part in the l.h.s. is due to $(1 / 2)\left[P_{c a}-P_{c a}^{+}\right]=(i / 2) D_{c} J_{a}$. Since the axial current is a vector, we can rewrite the imaginary part of the last equation as [C.f. footnote ${ }^{7}$,

$$
(1 / 2) h_{\mu}^{b} \gamma_{a c b} \nabla_{c} \mathcal{J}_{a}=(1 / 4) \eta^{a b}\left(\nabla_{\mu} h_{a}^{v}\right) h_{b}^{\lambda}\left(\nabla_{\nu} \mathcal{J}_{\lambda}-\nabla_{\lambda} \mathcal{J}_{v}\right)=0, \quad\left\{=2 m g \mathcal{S} \mathcal{W}_{b}\right\}
$$

which is dual to Equation (4.16). The skew-symmetric Hermitian part, $\left(P_{c a}+P_{c a}^{+}\right)-\left(P_{a c}+P_{a c}^{+}\right)$, must vanish since the r.h.s. of Equation (4.20) is an imaginary quantity. Since $\epsilon^{\text {stua }} j_{a}=i \psi^{+} \rho_{3} \alpha^{s} \rho_{1} \alpha^{t} \rho_{1} \alpha^{u} \psi,(s, t, u, \neq)$, this yields the equation,

$$
\gamma_{a c b}\left[\psi^{+} \rho_{3} \alpha_{a} \vec{D}_{c} \psi-\psi^{+} \overleftarrow{D}_{c}^{+} \alpha_{a} \rho_{3} \psi-\psi^{+} \rho_{3} \alpha_{c} \vec{D}_{a} \psi+\psi^{+} \overleftarrow{D}_{a}^{+} \alpha_{c} \rho_{3} \psi\right]=2 \gamma_{a c b} \epsilon^{a c u t} D_{u} j_{t}=4 \gamma_{a c b}^{\star} D_{a} j_{c}=0 .
$$

which is similar to Equation (4.16) and dual to Equation (4.15).

4. A full set of prerequisites for the covariance. Considered together, Equations (4.15) and (4.22) constitute a linear system of eight equations for the six unknowns, $\nabla_{a} j_{b}-\nabla_{b} j_{a}$. In general, the rank of its matrix equals 6 . Therefore, it can only have a trivial solution. Since $\nabla_{a} j_{b}$ are the invariants of a true tensor, $\nabla_{\mu} j_{v}$, we have the tensor equation,

$$
\nabla_{\mu} j_{v}-\nabla_{v} j_{\mu}=0
$$

Equations (4.16) and (4.21) constitute the system of 8 equations for 10 unknown quantities, $\nabla_{[v} \mathcal{J}_{\lambda]}$ and $\mathcal{W}_{\mu}$. These equations also explicitly depend on a choice of the auxiliary field of tetrad $\boldsymbol{h}_{a}(x)$, which is unacceptable. Insisting on independence as a physical (and then mathematical) requirement and realizing that $\mathcal{W}$ does not exist as a physical field, we must put $\mathcal{W}_{a}=0^{10}$. Then we have the system of 8 homogeneous equations for only 6 unknowns $\nabla_{[v} \mathcal{J}_{\lambda]}$ with a trivial solution,

$$
\nabla_{v} \mathcal{J}_{\lambda}-\nabla_{\lambda} \mathcal{J}_{v}=0
$$

which is similar to Equations (4.23) that we had for the vector current.

More identities are readily obtained along the same guidelines as Equation (4.14). Namely, duplicating (4.12)(4.14), we compute $D_{c}\left[\left(\psi^{c}\right)^{+} \alpha^{a} D_{a} \psi\right]$ and $D_{c}\left[\left(\psi^{c}\right)^{+} \overleftarrow{D}_{a}^{c+} \alpha^{a} \psi\right]$ directly and using equations of motion. Adding up the results we obtain that

$$
\gamma_{a d c} D_{a}\left[\left(\psi^{c}\right)^{+} \alpha_{d} \psi\right]=\gamma_{a d c} D_{a} \Lambda_{d}^{(+)}=0 .
$$

Computing in the same way the dual quantities, $D_{c}\left[\left(\psi^{c}\right)^{+} \rho_{3} \alpha^{a} D_{a} \psi\right]$ and $D_{c}\left[\left(\psi^{c}\right)^{+} \overleftarrow{D}_{a}^{c+} \rho_{3} \alpha^{a} \psi\right]$, we end up with

$$
\stackrel{\star}{\gamma}_{a d c} D_{a}\left[\left(\psi^{c}\right)^{+} \alpha_{d} \psi\right]=\stackrel{\star}{\gamma}_{a d c} D_{a} \Lambda_{d}^{(+)}=0,
$$

which once again is a system of 8 equations for six unknowns with only a trivial solution. Since $\gamma_{\text {adc }}$ is skewsymmetric in the first two indices and is not zero, we arrive at

which, by virtue of (4.6), results in

$$
D_{a} \Lambda_{b}^{( \pm)}-D_{b} \Lambda_{a}^{( \pm)}=0
$$

$$
\nabla_{\mu} \Theta_{\nu}-\nabla_{\nu} \Theta_{\mu}=-2 e\left(A_{\mu} \Phi_{v}-A_{\nu} \Phi_{\mu}\right), \quad \nabla_{\mu} \Phi_{v}-\nabla_{\nu} \Phi_{\mu}=+2 e\left(A_{\mu} \Theta_{v}-A_{\nu} \Theta_{\mu}\right) .
$$

The differential identities (4.15), (4.23) and (4.28) for the Dirac currents are written down in the covariant tensor form and can be transformed further into tetrad representation with respect to any tetrad. Therefore, it is indeed possible to overcome the Cartan's veto [C.f. footnote 4] relying on the second reservation in Cartan's statement.

\footnotetext{
${ }^{10}$ This accomplishes the proof of the statement outlined in the footnote7.
} 


\section{Dirac Field and Congruences of Curves}

Each of four linear partial differential equations, $\partial_{A} f=e_{A}^{\mu} \partial_{\mu} f=0$, determine a congruence of lines because it is equivalent to the system of three ODEs for unknown $x^{\mu}, \mathrm{d} x^{\mu} / e_{A}^{\mu}(x)=\mathrm{d} S^{A}, \mu=0,1,2,3$. The question is whether two or three of these PDEs can be solved together (if they form a complete system). The answer is encoded in the properties of the rotation coefficients $\omega_{A B}^{C}$ of the orthogonal net of the tetrad $\boldsymbol{e}_{A}$. These are not given a priori, but it is possible to find them as dynamic quantities. This is an immediate goal of this section. Technically, we will rely only on Equation (3.15),

$$
\nabla_{\mu}\left(\mathcal{R} e_{B}^{v}\right)=e_{B}^{v} \partial_{\mu} \mathcal{R}+\mathcal{R} \nabla_{\mu} e_{B}^{v}, \quad \nabla_{\mu} e_{B}^{v}=e_{\mu}^{D} \omega_{B D}^{A} e_{A}^{v}, \quad \nabla_{\mu} e_{B}^{\mu}=\omega_{B A}^{A}=\omega_{B 00}-\omega_{B 11}-\omega_{B 22}-\omega_{B 33} .
$$

\subsection{Vector Current and Timelike Congruence}

To analyze the lines of the vector current, the two obtained earlier equations, (4.3) and (4.23),

$$
\nabla_{\mu} j_{v}-\nabla_{v} j_{\mu}=0, \quad \nabla_{\mu} j^{\mu}=0,
$$

must be examined together. When the invariant density of the Dirac (spinor) matter is positive, $\mathcal{R}=\sqrt{j^{2}}>0$, the vector field $j^{\mu}(x)$ is strictly timelike; its tangent unit vector is $e_{[0]}^{\mu}(x), j^{\mu}=\mathcal{R} e_{[0]}^{\mu}$. Therefore, Equation (4.23) becomes

$$
\nabla_{\mu} e_{v}^{[0]}-\nabla_{\nu} e_{\mu}^{[0]}+e_{v}^{[0]} \partial_{\mu} \ln \mathcal{R}-e_{\mu}^{[0]} \partial_{v} \ln \mathcal{R}=0 .
$$

Contracting this equation with $e_{A}^{v} e_{B}^{\mu}, A, B=1,2,3$ and using Equation (5.1) we find that

$$
\omega_{0 A B}-\omega_{0 B A}=0, \quad A, B=1,2,3 \text {, }
$$

which is a necessary and sufficient condition for the congruence $e_{[0]}^{\mu}$ to be normal [17] [18]. Namely, there exists such a function, $\tau(x)$, that the vector field $e_{\mu}^{[0]}(x)$ is orthogonal to the family of surfaces $\tau(x)=$ const ,

$$
\partial_{\mu} \tau(x)=f(x) e_{\mu}^{[0]}(x), \quad \mathrm{d} \tau=\partial_{\mu} \tau \mathrm{d} x^{\mu}=f e_{\mu}^{[0]} \mathrm{d} x^{\mu}=f \mathrm{~d} S^{0},
$$

where $\tau(x)$ satisfies the complete system of three equations, $e_{A}^{\mu}(x) \partial_{\mu} \tau(x)=0, A=1,2,3$, and $f(x)$ is a coordinate scalar. Contracting Equation (5.3) with $e_{[0]}^{v}$ we get

$$
\partial_{\mu} \ln \mathcal{R}=e_{\mu}^{[0]} \partial_{[0]} \ln \mathcal{R}-\omega_{\text {B00 }} e_{\mu}^{(B)},
$$

where $\partial_{A} \ln \mathcal{R}=e_{A}^{\mu} \partial_{\mu} \ln \mathcal{R}=\partial \ln \mathcal{R} / \partial S^{A}$ is the derivative in the direction of the arc $S^{A}$. Contraction of Equation (5.3) with $e_{[0]}^{\nu} e_{A}^{\mu}$ yields

$$
\partial_{A} \ln R=-\omega_{A 00}, \quad A=1,2,3,
$$

which indicates that congruences of lines, defined by the system of equations, $d x^{\mu} / d S^{[0]}=e_{[0]}^{\mu}$, must experience permanent bending (acceleration) whenever the invariant density $\mathcal{R}(x)$ of the Dirac field is not uniformly distributed. The spatial gradient of $\mathcal{R}(x)$ cannot vanish for any localized state.

Additional information can be extracted from Equation (4.3), $\nabla_{v}\left(\mathcal{R} e_{[0]}^{v}\right) \equiv \partial_{[0]} \mathcal{R}+\mathcal{R} \nabla_{\nu} e_{[0]}^{v}=0$. Then, by definition,

$$
\nabla_{\nu} e_{[0]}^{v}=\omega_{0 A}^{A}=\omega_{101}+\omega_{202}+\omega_{303}=-\partial_{[0]} \ln \mathcal{R} \text {. }
$$

Hence, we can rewrite (5.6) as

$$
\partial_{\mu} \ln \mathcal{R}=-e_{\mu}^{[0]} \eta^{A B} \omega_{0 A B}-\omega_{B 00} e_{\mu}^{B},
$$

which shows that the r.h.s. of Equation (5.9), which contains only geometric objects, is a component of a gradient. Together with condition (5.4) this constitutes a necessary and sufficient condition that the function $\tau(x)$ defined by Equation (5.5) is an harmonic function [17],

$$
\square \tau=g^{\mu v} \nabla_{\mu} \nabla_{\nu} \tau=0
$$

The parameter $\tau^{*}$ of $\tau(x)=\tau^{*}=$ const is the definition of the world time. For the harmonic function, 
$\tau(x)$, the conditions of integrability for system (5.5) of partial differential equations reads as [17]

$$
\partial_{\mu} \ln f=-e_{\mu}^{[0]} \eta^{A B} \omega_{0 A B}-\omega_{B 00} e_{\mu}^{(B)} .
$$

Comparing it with (5.9) we find that $f(x)=\mathcal{R}$, so that the world time $\tau$ and the "proper time" $S^{[0]}$ are related by

$$
\mathrm{d} \tau=\mathcal{R} \mathrm{d} S^{[0]}
$$

Furthermore, since $f(x)=\mathcal{R}$ and system possesses the proper time, we can rewrite Equation (5.9) as $j_{\mu}(x)=\partial_{\mu} \tau(x)$, which could have been inferred directly from Equation (4.15). Then, the harmonic nature of $\tau(x)$ immediately follows from the current conservation, $\nabla_{\mu} j^{\mu}=0$. Since $\mathrm{d} \tau$ is the total differential and the vector current $\boldsymbol{j}$ belongs, in fact, to the principal manifold $\mathbb{M}$, so does the interval of the world time $\tau$,

$$
\tau_{2}-\tau_{1}=\int_{x\left(\tau_{1}\right)}^{x\left(\tau_{2}\right)} j_{\mu}(x) \mathrm{d} x^{\mu}\left(=\int \mathcal{R} \mathrm{d} S^{[0]}\right),
$$

and this interval does not depend on the path of integration (the time variable $\tau$ is a holonomic coordinate).

Now, we can draw the major conclusion: The proper time, $S^{[0]}$, flows more slowly than the world time, $\tau$, whenever Dirac matter has a magnified density. Because of the wave nature of the Dirac field, its localization is inevitable. Since the congruence $e_{[0]}^{\mu}$ appeared to be normal, the hypersurfaces $\tau(x)=\tau^{*}=$ const represent space at different times $\tau^{*}$. The states can be considered stationary only with respect to $\tau$; one can hope to find them only after replacing $i \partial / \partial S^{0}$ by $i \mathcal{R} \partial / \partial \tau$ in the operator of energy!

\subsection{Axial Current and Radial Congruence}

Here, we have to deal with the system of equations,

$$
\nabla_{\nu} \mathcal{J}_{\lambda}-\nabla_{\lambda} \mathcal{J}_{v}=0, \quad \nabla_{\nu} \mathcal{J}^{v}=2 m \mathcal{P},
$$

which is similar to Equations (5.2) that we had for the vector current. The only difference is that the axial current has a source $2 m \mathcal{P}$. Since there is no flux of vector current in this direction (the amount of matter inside a closed surface remains the same), we associate the radial direction $e_{[3]}^{\mu}(x)$ with the axial current, $J^{\mu}=\mathcal{R} e_{[3]}^{\mu}$. Next, observe that by virtue of the Fierz identity (2.3), $\mathcal{R}^{2}=\mathcal{S}^{2}+\mathcal{P}^{2}$, we can parameterize, $\mathcal{S}=\mathcal{R} \cos \mathcal{Y}$, $\mathcal{P}=\mathcal{R} \sin \mathcal{Y}$. Then the second Equation (5.13) takes form

$$
\nabla_{\mu} e_{[3]}^{\mu}+e_{[3]}^{\mu} \partial_{\mu} \ln \mathcal{R}=2 m \mathcal{P} / \mathcal{R}=2 m \sin \mathcal{Y} .
$$

On the one hand, by definition, $\nabla_{\mu} e_{[3]}^{\mu}=\eta^{A B} \omega_{3 A B}=\omega_{300}-\omega_{311}-\omega_{322}$. On the other hand, according to Equation (5.7), we have $e_{[3]}^{\mu} \partial_{\mu} \ln \mathcal{R}=\partial_{[3]} \ln \mathcal{R}=-\omega_{300}$. Substituting these expressions into Equation (5.14) we obtain an important relation,

$$
\omega_{131}+\omega_{232}=2 m \sin \mathcal{Y} .
$$

The first of Equations (5.13), being contracted with $e_{A}^{\mu} e_{B}^{v}$, yields

$$
\omega_{3 A B}-\omega_{3 B A}=0, \quad A, B=0,1,2, A \neq B,
$$

so that the congruence of lines $\boldsymbol{e}_{[3]}$ is normal and there exists such a family of hypersurfaces $\rho(x)=\rho^{*}=$ const that

$$
\partial_{\mu} \rho(x)=n(x) e_{\mu}^{[3]}(x)
$$

where $\rho(x)$ satisfies the complete system of three equations, $e_{A}^{\mu}(x) \partial_{\mu} \rho(x)=0, A=0,1,2$, and $n(x)$ is a coordinate scalar. In the same way as before [cf. (5.6), (5.7)], contracting the first of Equations (5.13) with $e_{[3]}^{v}$ and $e_{A}^{\mu} e_{[3]}^{v}$, we will get

$$
-\partial_{\mu} \ln \mathcal{R}=e_{[3] \mu} \partial_{[3]} \ln \mathcal{R}-\omega_{\text {Аз3 }} e_{\mu}^{A}, \partial_{A} \ln \mathcal{R}=-\omega_{3 A 3}, \quad A=0,1,2,
$$

and this is compatible with the condition for integrability, $-\partial_{\mu} \ln n=e_{[3] \mu} \partial_{[3]} \ln n-\omega_{A 33} e_{\mu}^{A}$, of the system (5.17) only when $n(x) / \mathcal{R}(x)=$ const . Next, we may compute the second derivative of $\rho$. Using Equation (5.7) and Equation (5.27) below, we arrive at 


$$
g^{\mu \nu} \nabla_{\mu} \nabla_{v} \rho=-\nabla_{\mu}\left(n e_{[3]}^{\mu}\right)=n\left[\omega_{3 A}^{A}+\partial_{[3]} \ln n\right]=n\left[-3 \partial_{[3]} \ln \mathcal{R}+\partial_{[3]} \ln n\right]=n \partial_{[3]} \ln \left(n / \mathcal{R}^{3}\right)
$$

From here we find that if $n(x)=\mathcal{R}(x)$, then $\rho(x)$ is the solution of an inhomogeneous wave equation,

$$
\square \rho=g^{\mu \nu} \nabla_{\mu} \nabla_{v} \rho=2 m \mathcal{P}, \partial_{\mu} \rho=\mathcal{R} e_{\mu}^{[3]}=-\mathcal{J}_{\mu}, e_{A}^{\mu} \partial_{\mu} \rho=\mathcal{R} \delta_{A}^{3},
$$

for the "potential” $\rho$ with the source density proportional to the mass parameter $m$ of the Dirac equation and pseudoscalar density $\mathcal{P}$ (in static limit, it becomes the Poisson equation). Not surprisingly, this source is equal to the derivative of the invariant density in the direction of the axial current. If the invariant density was not changing in a "radial direction", the whole idea of a localized object would be vague. Similarly to (5.5) and (5.11), we have

$$
\mathrm{d} \rho=\mathrm{d} x^{\mu} \partial_{\mu} \rho=\mathcal{R} e_{\mu}^{[3]} \mathrm{d} x^{\mu}=\mathcal{R} \mathrm{d} S^{[3]} .
$$

From here, we conclude that the differential form $\mathcal{J}_{\mu} \mathrm{d} x^{\mu}$ is integrable and the "radial distance",

$$
\rho_{2}-\rho_{1}=\int_{x\left(\rho_{1}\right)}^{x\left(\rho_{2}\right)} \mathcal{J}_{\mu}(x) \mathrm{d} x^{\mu}\left(=\int \mathcal{R} \mathrm{d} S^{[3]}\right),
$$

does not depend on the integration path (the coordinate variable $\rho$ is holonomic).

\subsection{Congruences of the Angular Arcs}

Here, we must deal with four equations (4.6) and (4.28). Taking $e_{[1]}^{\mu}=\Theta^{\mu}(x) / \mathcal{R}, e_{[2]}^{\mu}=\Phi^{\mu}(x) / \mathcal{R}$ (an alternative choice with $e_{[1]}^{\mu} \leftrightarrow e_{[2]}^{\mu}$ will be discussed later), starting from Equation (4.6), and duplicating the derivation of Equation (5.8) we arrive at the equations,

$$
\omega_{212}+\omega_{313}=-2 e A_{[2]}, \quad \omega_{121}+\omega_{323}=+2 e A_{[1]} .
$$

Since by the second Equation (5.18) we have $\omega_{313}=-\partial_{[1]} \ln \mathcal{R}$ and $\omega_{323}=-\partial_{[2]} \ln \mathcal{R}$, these equations completely define $\omega_{212}$ and $\omega_{121}$,

$$
\omega_{212}=\partial_{[1]} \ln \mathcal{R}-2 e A_{[2]}, \quad \omega_{121}=\partial_{[2]} \ln \mathcal{R}+2 e A_{[1]} .
$$

Putting further in Equations (4.28) $\Theta_{\mu}=\mathcal{R} e_{[1]}^{\mu}$ and $\Phi_{\mu}=\mathcal{R} e_{[2]}^{\mu}$, and duplicating the scheme of Equation (5.3)-(5.7), we obtain,

$$
\begin{aligned}
& \omega_{1 B A}-\omega_{1 A B}=-2 e\left(A_{A} \eta_{2 B}-A_{B} \eta_{2 A}\right), A, B \neq 1 ; A \neq B, \\
& -\omega_{1 A 1}-\partial_{A} \ln \mathcal{R}=2 e A_{[1]} \eta_{2 A}, A=0,2,3, \\
& \omega_{2 B A}-\omega_{2 A B}=2 e\left(A_{A} \eta_{1 B}-A_{B} \eta_{1 A}\right), A, B \neq 2 ; A \neq B, \\
& -\omega_{2 A 2}-\partial_{A} \ln \mathcal{R}=-2 e A_{[2]} \eta_{1 A}, A=0,1,3 .
\end{aligned}
$$

Giving index $A$ in Equations (5.24) and (5.26) all possible values, we get the following constraints,

$$
\begin{aligned}
& \omega_{101}=\omega_{202}=-\partial_{[0]} \ln \mathcal{R}, \omega_{[3]}=\omega_{232}=-\partial_{[3]} \ln \mathcal{R}=m \sin \mathcal{Y} ; \\
& \omega_{212}=-2 e A_{[2]}-\partial_{[1]} \ln \mathcal{R}, \omega_{[2]}=2 e A_{[1]}-\partial_{[2]} \ln \mathcal{R} .
\end{aligned}
$$

Equations (5.28) and (5.22) are mutually compatible only when $\partial_{[1]} \ln \mathcal{R}=\partial_{[2]} \ln \mathcal{R}=0$, and

$$
\omega_{010}=\omega_{313}=\partial_{[1]} \ln \mathcal{R}=0, \quad \omega_{020}=\omega_{323}=\partial_{[2]} \ln \mathcal{R}=0,
$$

i.e., when the vectors of the geodesic curvature $\omega_{0 A 0}$ and $\omega_{3 A 3}$ of the congruences [0] and [3] of the vector and axial currents have no projections on the lines of the congruences [1] and [2] of the charged currents.

\footnotetext{
${ }^{11}$ Having no metric, we assume here geodesic of an affine space, i.e. such a line $x^{\mu}(s)$ that its tangent vector, $p^{\mu}(s)=\mathrm{d} x^{\mu} / \mathrm{d} s$, is parallel transported (with respect to an affine connection $\Gamma_{\lambda \sigma}^{\mu}$ (3.14)) along the line, $\mathrm{d} p^{\mu} / \mathrm{d} s \propto p^{\mu}$. In our particular case of the tetrad vector $\boldsymbol{e}_{3}$, this amounts to $\nabla_{3} \boldsymbol{e}_{3}=\omega_{33}^{A} \boldsymbol{e}_{A} \propto \omega_{33}^{3} \boldsymbol{e}_{3} \equiv 0$.
} 
Together with the previously obtained Equations (5.8), (5.18) and (5.22), they give all $\omega_{A B A}$ in terms of derivatives of the invariant density and electromagnetic potentials. Namely, since $\omega_{303}=-\partial_{[0]} \ln \mathcal{R}$, we also have $\omega_{101}+\omega_{202}=0$, which together with the first Equation (5.27) entails that

$$
\partial_{[0]} \ln \mathcal{R}=0, \quad \omega_{3 A 3}=0, \quad A=0,1,2 .
$$

The second of these equations means that the congruence [3] is geodesic ${ }^{11}$. Quite remarkably, this conclusion about static character of the configuration that satisfies Dirac equations of motion is reached only after all the differential identities are considered together. The additional constraints that follow from Equations (5.23) and (5.25), when indices $A$ and $B$ are given all possible values, are as follows,

$$
\begin{aligned}
& \omega_{130}=\omega_{103}, \omega_{230}=\omega_{203}, \omega_{120}-\omega_{102}=2 e A_{[0]}, \\
& \omega_{123}-\omega_{132}=2 e A_{[3]}, \quad \omega_{231}-\omega_{213}=2 e A_{[3]} .
\end{aligned}
$$

Combined with the previous results (Equation (5.4), particularly) they yield,

$$
\begin{aligned}
& \omega_{120}=2 e A_{10]}, \omega_{012}=\omega_{021}=0, \\
& \omega_{3 A B}=-\omega_{3 B A} ; A, B=0,1,2 ; A \neq B .
\end{aligned}
$$

The last of these equations is the necessary and sufficient condition for the congruences of lines $\boldsymbol{e}_{[0]}, \boldsymbol{e}_{[1]}$ and $\boldsymbol{e}_{[2]}$ being canonical of the congruence $\boldsymbol{e}_{[3]}$ [18]. This property appears to be yet another consequence of the Dirac equation of motion, which thus guarantees that the orthogonal tetrad is Fermi-transported. Finally, comparing Equations (5.16) and (5.34) we find that

$$
\omega_{3 A B}=0, \quad A, B=0,1,2 ; A \neq B .
$$

\subsection{Summary-Coefficients of Rotations That Completely Define the Matter-Induced Affine Geometry}

By now, we have succeeded to find simple expressions for all coefficients $\omega_{A B C}$ of rotation of the basis $\boldsymbol{e}_{A}$ of the normalized Dirac currents. This is the last step in the design of the matter-induced affine geometry. From this point, one can rely on the common tools of the differential geometry. We can divide the not vanishing components of $\omega_{A B C}$ into two distinct groups:

1) Five geodesic curvatures ( the $\omega_{A B C}$ with only two distinct indices),

$$
\begin{aligned}
& \omega_{030}=-\omega_{131}=-\omega_{232}=-m \mathcal{P} / \mathcal{R}=-m \sin \mathcal{Y}, \\
& \omega_{121}=+2 e A_{11]}, \quad \omega_{212}=-2 e A_{[2]} .
\end{aligned}
$$

2) Only two of the $\omega_{A B C}$ with all three different indices are nonzero. These are

$$
\omega_{120}=-\omega_{210}=2 e A_{[0]}, \quad \omega_{123}=-\omega_{213}=2 e A_{[3]}
$$

3) The coefficients $\omega_{A B C}$, which depend on the potential $A_{D}$, are of the same form

$$
\omega_{12 D}=2 e A_{D} \text {, }
$$

so that presence of electromagnetic field causes rotation of the Dirac tetrad in the (12) - tangent plane. This inter-action makes it impossible, in general, to match Dirac equation with the all-orthogonal system of hypersurfaces $^{12}$.

It is essential that the only directional derivative that survived all constrains is $\partial_{[3]} \mathcal{R}$, and even it can be

\footnotetext{
${ }^{12}$ Keeping up with the promise given in Section 3, we compute, following Equation (3.10), the coefficients of rotation $\gamma_{a b d}$ of the basis $\boldsymbol{h}_{a}$.

$$
\gamma_{a b d}=\left[\omega_{12 D} V_{d}^{D} V_{a}^{[1]} V_{b}^{[2]}+V_{a}^{[0]} V_{b}^{[3]} V_{d}^{[0]} \omega_{030}+V_{a}^{[1]} V_{b}^{[3]} V_{d}^{[1]} \omega_{131}+V_{a}^{[2]} V_{b}^{[3]} V_{d}^{[2]} \omega_{232}\right]-(b \leftrightarrow a) .
$$

Using Equations (5.36)-(5.37) and employing Equation (2.5) as, $V_{a}^{[0]} V_{d}^{[0]}-V_{a}^{[1]} V_{d}^{[1]}-V_{a}^{[2]} V_{d}^{[2]}=\eta_{a d}+V_{a}^{[3]} V_{d}^{[3]}$, we obtain,

$$
\gamma_{a b d}(\psi)=2 e A_{d}\left(V_{a}^{[1]} V_{b}^{[2]}-V_{b}^{[1]} V_{a}^{[2]}\right)+m \sin \mathcal{Y} \cdot\left(V_{a}^{[3]} \eta_{b d}-V_{b}^{[3]} \eta_{a d}\right) .
$$


expressed via pseudoscalar density. Therefore, the practical computation of the connection $\omega_{A B C}$ does not require any reference to a coordinate background. The congruence of integral lines of the vector field $\boldsymbol{e}_{3}$ is both normal and geodesic. This is the only geodesic of the principal manifold $\mathbb{M}$, and it is inherited by the hypersurfaces of the constant world time. The congruences $\boldsymbol{e}_{0}, \boldsymbol{e}_{1}, \boldsymbol{e}_{2}$ constitute a canonical system with respect to the congruence $\boldsymbol{e}_{3}$. Therefore the entire tetrad is Fermi-transported along the the lines of the radial congruence $\boldsymbol{e}_{3}$. Equations (5.36)-(5.39) assume a localized configuration with maximum of invariant density in its interior and a naturally right-handed spatial trihedron $\left(\boldsymbol{e}_{1}, \boldsymbol{e}_{2}, \boldsymbol{e}_{3}\right)$. If there is a minimum, then the signs of tetrad components $A_{B}$ in coefficients of rotation (5.36)-(5.37) (and only there!) must be reverted.

\section{Coordinate Surfaces and Coordinate Lines of the Dirac Field}

Below, we attempt to find the submanifolds of the physical manifold $\mathbb{M}$, which can be mapped onto coordinate surfaces of the arithmetic $\mathbb{R}^{4}$. An advance knowledge of these surfaces will be critical for finding the autolocalized Dirac waveforms and then understanding their shape and internal field structure. If we denote the differential operators $e_{A}^{\mu} \partial_{\mu}$ as $\partial_{[A]}$ and introduce, for the sake of brevity, $Q \equiv \partial_{[3]} \ln \mathcal{R}=-m \mathcal{P} / \mathcal{R}=-m \sin \mathcal{Y}$, then an explicit calculation according to the second Equation (3.23),

$$
\left(\partial_{A} \partial_{B}-\partial_{B} \partial_{A}\right) \psi=-\left(\omega_{A B}^{D}-\omega_{B A}^{D}\right) \partial_{D} \psi \equiv C_{A B}^{D} \partial_{D} \psi
$$

yields the following expressions for the Poisson brackets,

$$
\begin{aligned}
& \left(\partial_{[0]} \partial_{[3]}-\partial_{[3]} \partial_{[0]}\right) f=-Q \cdot \partial_{[0]} f, \\
& \left(\partial_{[0]} \partial_{[1}-\partial_{[1]} \partial_{[0]}\right) f=-2 e A_{[0]} \partial_{[2]} f, \\
& \left(\partial_{[0]} \partial_{[2]}-\partial_{[2]} \partial_{[0]}\right) f=2 e A_{[0]} \cdot \partial_{[1]} f, \\
& \left(\partial_{[1]} \partial_{[2]}-\partial_{[2]} \partial_{[1]}\right) f=2 e A_{[2]} \cdot \partial_{[2]} f+2 e A_{[1]} \cdot \partial_{[1]} f, \\
& \left(\partial_{[3]} \partial_{[1]}-\partial_{[1]} \partial_{[3]}\right) f=Q \cdot \partial_{[1]} f-2 e A_{[3]} \cdot \partial_{[2]} f, \\
& \left(\partial_{[3]} \partial_{[2]}-\partial_{[2]} \partial_{[3]}\right) f=Q \cdot \partial_{[2]} f+2 e A_{[3]} \cdot \partial_{[1]} f .
\end{aligned}
$$

These expressions allow one to completely explore properties not only of the individual congruences and 3-d hypersurfaces but also of the 2-d surfaces. The latter is imperative as long as we aim at (and already have a hint of) dynamic localization of the Dirac field into finite-sized objects.

Some immediate observations are in order. Equations (6.1) are nothing but differential identities that express the integrability of the directional derivatives. From equations of motion we know that $\partial_{A} \mathcal{R}=0$ for $A=0,1,2$ and $\partial_{[3]} \mathcal{R}=-m \mathcal{P}$. Let us take in Equation (6.1) $f=\mathcal{R}$ and use Equations (5.29) and (5.30). Then from Equations (6.1.e,f) we have $\partial_{[1]} \mathcal{P}=0$ and $\partial_{[2]} \mathcal{P}=0$, while Equation (6.1.a) yields $\partial_{[0]} \mathcal{P}=0$. Thus, we have even more constraints,

$$
\partial_{A} \mathcal{R}=\partial_{A} \mathcal{S}=\partial_{A} \mathcal{P}=\partial_{A} \mathcal{Y}=0, \quad A=0,1,2
$$

At any point $\mathrm{P}$ of the principal manifold $\mathbb{M}$ all the scalars change only in the direction $\boldsymbol{e}_{3}$ of the axial current, and the rate of this change is determined by the product $m \mathcal{P}$.

\subsection{Integrable Subsystems and Coordinate Surfaces in $\mathbb{R}^{4}$}

Since we are aiming at the discovery of the localized solutions, a coordinate picture may become most appropriate, and it is useful to know in advance what the admissible coordinate net may look like. Solely for this purpose, we study here whether the congruences of the Dirac currents in $\mathbb{R}^{4}$ can form at least some of the four 3-d coordinate hypersurfaces and of the six 2-d coordinate surfaces. Once found, these surfaces will be studied in detail as submanifolds embedded into $\mathbb{M}^{4}$ endowed with the connections identified above.

1. Hypersurfaces $\boldsymbol{S}_{(123)}$ and $\boldsymbol{S}_{(\mathbf{1 2 0 )})}$. From visual inspection of the Poisson brackets (6.1), among the four equations, $e_{A}^{\mu} \partial_{\mu} f=0$, there are two integrable systems of three equations that define two hypersurfaces and two integrable system of two equations that define two surfaces in the coordinate space $\mathbb{R}^{4}$. Namely, three com- 
mutators between the $\partial_{[1]}, \partial_{[2]}$ and $\partial_{[3]}$ [Equations $\left.(6.1 \mathrm{~d}, \mathrm{e}, \mathrm{f})\right]$ are the linear combinations of these operators alone. Therefore, the function $\tau(x)=\tau^{*}=$ const (as well as any function $f(\tau)$ ) is the first integral of the complete (Jacobian) system of three equations,

$$
e_{[1]}^{\mu} \partial_{\mu} \tau=0, e_{[2]}^{\mu} \partial_{\mu} \tau=0 \text { and } e_{[3]}^{\mu} \partial_{\mu} \tau=0 .
$$

The parameter $\tau^{*}$ enumerates the family of hypersurfaces $S_{(123)}$, which are spanned by the streamlines of the vector fields $e_{[1]}^{\mu}, e_{[2]}^{\mu}$ and $e_{[3]}^{\mu}$ and have $e_{\mu}^{[0]}$ as the normal. Equations $(6.1 \mathrm{~b}, \mathrm{c}, \mathrm{d})$ indicate that three equations,

$$
e_{[1]}^{\mu} \partial_{\mu} \rho=0, e_{[2]}^{\mu} \partial_{\mu} \rho=0 \text { and } e_{[0]}^{\mu} \partial_{\mu} \rho=0 \text {, }
$$

also constitute an integrable system with a first integral $\rho(x)=\rho^{*}=$ const (or any function $f(\rho)$ ); the latter represents hypersurfaces $S_{(120)}$ of the constant "radius" $\rho$ when $\mathcal{R}^{2}>0$. These are spanned by the integral lines of the vector fields $e_{[1]}^{\mu}, e_{[2]}^{\mu}$ and $e_{[0]}^{\mu}$ and have $e_{\mu}^{[3]}$ as the spacelike normal.

2. Surfaces $\boldsymbol{S}_{(12)}$ and $\boldsymbol{S}_{(03)}$. Next, by Equation (6.1 d) the system of equations

$$
e_{[1]}^{\mu}(x) \partial_{\mu} \vartheta=0, \quad e_{[2]}^{\mu}(x) \partial_{\mu} \vartheta=0
$$

is integrable. Its two first integrals, $\theta_{1}(x)=c_{1}$ and $\theta_{2}(x)=c_{2}$, determine a two-dimensional surface $S_{(12)}$ spanned by the streamlines of the vector fields $e_{1}^{\mu}$ and $e_{2}^{\mu}$ having the normal vectors $v_{\mu}=\partial_{\mu} \vartheta=c_{0} e_{\mu}^{0}+c_{3} e_{\mu}^{3}$. The first integrals of the system (6.5) are known because both of its equations are satisfied by $\vartheta(x)=\tau(x)=\tau^{*}$ and $\vartheta(x)=\rho(x)=\rho^{*}$. Once $\tau(x)$ and $\rho(x)$ are algebraically independent, these are the two first integrals of the system (5), and the 2-d surface $S_{(12)}$ is uniquely fixed by the values of constants $\tau^{*}$ and $\rho^{*}$, which enumerate the surfaces of a constant "radius" $\rho$ at a given "world time" $\tau$.

Finally, according to Equation (6.1 a) the commutator between $\partial_{[0]}$ and $\partial_{[3]}$ is proportional to $\partial_{[0]}$. Therefore, the system of equations

$$
e_{[0]}^{\mu}(x) \partial_{\mu} \varphi=0, e_{[3]}^{\mu}(x) \partial_{\mu} \varphi=0
$$

is integrable. It has two first integrals, $\phi_{1}(x)=C_{1}$ and $\phi_{2}(x)=C_{2}$, which determine a two-dimensional surface $S_{(03)}$ spanned by the streamlines of the vector fields $e_{[0]}^{\mu}$ and $e_{[3]}^{\mu}$. The two normal vectors $n_{\mu}=\partial_{\mu} \phi$ of these surfaces are the linear combinations $c_{1} e_{\mu}^{[1]}+c_{2} e_{\mu}^{[2]}$. One of the first integrals of the second Equation (6.6) is $\varphi(x)=\tau(x)$, i.e. we have $e_{[3]}^{\mu}(x) \partial_{\mu} \tau(x)=0$. Also, one of the first integrals of the first Equation (6.6) is $\varphi(x)=\rho(x)$, i.e. $e_{[0]}^{\mu}(x) \partial_{\mu} \rho(x)=0$. Since the congruences of integral lines of the fields $e_{[0]}^{\mu}$ and $e_{3}^{\mu}$ are normal- (cf. Section 5), we have $\partial_{\mu} \tau(x)=\mathcal{R} e_{[0] \mu}(x)$ and $e_{[0]}^{\mu}(x) \partial_{\mu} \tau(x)=\mathcal{R}$, as well as $\partial_{\mu} \rho(x)=\mathcal{R} e_{[3] \mu}(x)$ and $e_{[3]}^{\mu}(x) \partial_{\mu} \rho(x)=-\mathcal{R}$. In terms of the new independent variables, $\tau=\tau\left(x^{0}, x^{1}, x^{2}, x^{3}\right), x^{1}, x^{2}, \rho=\rho\left(x^{0}, x^{1}, x^{2}, x^{3}\right)$, the system (6.6) immediately acquires the normal (Jacobian) form,

$$
\begin{aligned}
& -\mathcal{R} \frac{\partial \varphi}{\partial \rho}+e_{[3]}^{1} \frac{\partial \varphi}{\partial x^{1}}+e_{[3]}^{2} \frac{\partial \varphi}{\partial x^{2}}=0 \\
& \mathcal{R} \frac{\partial \varphi}{\partial \tau}+e_{[0]}^{1} \frac{\partial \varphi}{\partial x^{1}}+e_{[0]}^{2} \frac{\partial \varphi}{\partial x^{2}}=0 .
\end{aligned}
$$

Its second equation is equivalent to the system of three ODEs,

$$
\frac{\mathrm{d} \rho}{0}=\frac{\mathrm{d} \tau}{\mathcal{R}}=\frac{\mathrm{d} x^{1}}{e_{[0]}^{1}}=\frac{\mathrm{d} x^{2}}{e_{[0]}^{2}},
$$

which has three first integrals, $\rho=\rho^{*}=$ const $, \phi_{1}=C_{1}, \phi_{2}=C_{2}$. In terms of the new independent variables, $\zeta^{0}=\tau, \zeta^{3}=\rho, \zeta^{1}=\phi_{1}, \zeta^{2}=\phi_{2}$, the system (6.7) reads as

$$
-\mathcal{R} \frac{\partial \varphi}{\partial \rho}+\theta^{1} \frac{\partial \varphi}{\partial \zeta^{1}}+\theta^{2} \frac{\partial \varphi}{\partial \zeta^{2}}=0, \mathcal{R} \frac{\partial \varphi}{\partial \tau}=0,
$$


where $\theta^{i}=\mathcal{R}\left(\partial \phi_{i} / \partial \rho\right)+e_{[3]}^{1}\left(\partial \phi_{i} / \partial x^{1}\right)+e_{[3]}^{2}\left(\partial \phi_{i} / \partial x^{2}\right)$. Since $\phi$ is independent of $\tau$, we have one PDE in three variables, which is equivalent to the system of two ODEs. The variables $\tau$ and $\rho$ form an orthogonal coordinate basis on every 2-d surface $S_{(03)}$ (enumerated by the values of $\zeta^{1}$ and $\zeta^{2}$ ).

\subsection{Coordinate Surfaces as Submanifolds in $\mathbb{M}$}

Conditions for simultaneous integrability of the PDEs for the streamlines of the Dirac currents prompted the existence of the (hyper)surfaces in $\mathbb{R}^{4}$ and, most importantly, in $\mathbb{M}$. Here, in order to understand their shape, we look at them as submanifolds of the principal manifold $\mathbb{M}$.

1. The method. For the sake of brevity, we will use the Latin capitals $H, \cdots, N=0,1,2,3$ to label the entire tetrad basis $\boldsymbol{e}_{H}$ (or $\boldsymbol{V}_{H}$ ). In the context of the current work this is the basis of the ambient space. The capitals $P_{t}=(P, \cdots, U)$ will label the tangent tetrad vectors of a 3-d or 2-d submanifold. The capitals $A_{n}=(A, \cdots, F)$ will be used to label the normal vectors. Then the induced metric of a submanifold is $g_{P Q}=g_{\mu \nu} e_{P}^{\mu} e_{Q}^{v}=\eta_{P Q}$ and, by virtue of definition (2.11), the first quadratic form of the surface $S_{(P \ldots)}$ is (pseudo)-Euclidean, $\mathrm{ds}{ }^{2}=$ $\eta_{P Q} \mathrm{~d} S^{P} \mathrm{~d} S^{Q}$.

Since we are interested in submanifolds that are spanned by the integral lines of the tetrad vectors, the Gauss and Weingarten decompositions of the covariant derivatives of tangent and normal (with respect to a submanifold) tetrad vectors immediately follow from Equations (3.2),

$$
\begin{aligned}
& \text { (Gauss) } \quad \bar{\nabla}_{R} V_{P}^{a}=\omega_{P R}^{H} V_{H}^{a}=\sum_{Q_{t}} \omega_{P R}^{Q} V_{Q}^{a}+\sum_{A_{n}} \omega_{P R}^{A} V_{A}^{a}, \\
& \text { (Weingarten) } \quad \bar{\nabla}_{R} V_{A}^{a}=\omega_{A R}^{H} V_{H}^{a}=\sum_{P_{t}} \omega_{A R}^{P} V_{P}^{a}+\sum_{B_{n}} \omega_{A R}^{B} V_{B}^{a},
\end{aligned}
$$

where all the $\omega$ 's listed in Equations (5.36)-(5.37) are known explicitly ${ }^{13}$. The first term, $\omega_{P R}^{Q}$, in the r.h.s. of the Gauss decomposition (6.10) is the connection of the intrinsic tangent space of the submanifold. The second term, $L_{P R}^{A}=\omega_{P R}^{A}$ (with two tangent and one normal indices), is the second fundamental form of the submanifold with respect to the normal $V_{A}$. The first term in Weingarten decomposition (6.11), $A_{A R}^{P}=\omega_{A R}^{P}=$ $-\eta_{A C} \eta^{Q P} \omega_{Q R}^{C}=-\eta_{A C} \eta^{Q P} L_{Q R}^{C}$, (the shape form with two tangent and one normal indices) is similar to the second fundamental form in (6.10); both account for the rotation of the tetrad in the (PA) plane when it is displaced in a tangent direction $\boldsymbol{e}_{R}$. The second term of Equation (6.11), $D_{A C ; R}=-D_{C A ; R}=\eta_{C B} \omega_{A R}^{B}$, with two normal and one tangent indices, is the covariant derivative of the normal components of a vector in a tangent direction of the submanifold. It accounts for the rotation of the $(A B)$ - plane of the two normals under infinitesimal displacement in tangent direction $\boldsymbol{e}_{R}$.

Now, since there is no question of how a submanifold is embedded into the ambient space with explicitly known tetrad vectors, we are in position to study the internal geometry of various coordinate surfaces, as submanifolds of the principal manifold $\mathbb{M}$. Besides the second fundamental form, we will use the Riemann curvature tensor in ambient space and in subspaces,

$$
R_{H K L}^{N}=\partial_{H} \omega_{L K}^{N}-\partial_{K} \omega_{L H}^{N}+\omega_{L M}^{N}\left(\omega_{H K}^{M}-\omega_{K H}^{M}\right)+\omega_{L H}^{M} \omega_{M K}^{N}-\omega_{L K}^{M} \omega_{M H}^{N}
$$

With these preliminaries, we are in the position to consider all subspaces on-by-one.

2. The hypersurface $\boldsymbol{S}_{(123)}$ represents space at a given time. It has three spacelike tangent vectors $\boldsymbol{V}_{P}$, $(P=1,2,3)$, and a single timelike normal vector $\boldsymbol{V}_{0}$. The coefficients of the single second fundamental form are $L_{12}^{0}=L_{23}^{0}=L_{31}^{0}=0$ and $L_{11}^{0}=L_{22}^{0}=L_{33}^{0}=-\partial_{[0]} \ln \mathcal{R}=0$. The second fundamental form, $I^{0}=L_{P Q}^{0} \mathrm{~d} S^{P} \mathrm{~d} S^{Q}$, is proportional to first fundamental form, $\mathrm{I}_{(123)}=\eta_{P Q} \mathrm{~d} S^{P} \mathrm{~d} S^{Q}=-\left(\mathrm{d} S^{1}\right)^{2}-\left(\mathrm{d} S^{2}\right)^{2}-\left(\mathrm{d} S^{3}\right)^{2}$ of the $S_{(123)}$,

$$
\|^{0}=\partial_{[0]} \ln \mathcal{R} \cdot\left[-\left(\mathrm{d} S^{1}\right)^{2}-\left(\mathrm{d} S^{2}\right)^{2}-\left(\mathrm{d} S^{3}\right)^{2}\right]=0 .
$$

Therefore, the $S_{(123)}$ is a totally umbilical submanifold ${ }^{14}$ with zero mean normal curvature $H=\partial_{[0]} \ln \mathcal{R}=0$. The latter means that $S_{(123)}$ is a totally geodesic submanifold; it inherits its sole geodesic $\boldsymbol{e}_{3}$ from the ambient $\mathbb{M}$. From the perspective of the ambient space, the hypersurface $S_{(123)}$ has no curvature, it is extrinsically flat.

\footnotetext{
${ }^{13}$ In mathematical literature the Gauss and Weingarten formulae are written down as $\bar{\nabla}_{X} Y=\nabla_{X} Y+h(X, Y)$ and $\bar{\nabla}_{X} \xi=-A_{\xi} X+D_{X} \xi$, respectively. Here, $X, Y$ are tangent and $\xi$ is normal to the submanifold.

${ }^{14} \mathrm{All}$ points of which are umbilical. A point is called umbilical if all principal curvatures at this point are equal.
} 
The extrinsic part vanishes together with the connections $\omega_{[0]}=\omega_{202}=-\omega_{303}=-\partial_{[0]} \ln \mathcal{R}=0$. The intrinsic Riemann curvature of the $S_{(123)}$ has six different (modulo sign) components; it is given by the terms of (6.12) with all indices in tangent space of the $S_{(123)}$,

$$
\begin{aligned}
& R_{1212}^{t}=2 e\left(\partial_{[1]} A_{[2]}-\partial_{[2]} A_{[1]}\right)-4 e^{2}\left(A_{[1]}^{2}+A_{[2]}^{2}\right)+Q^{2}=2 e F_{12}+Q^{2}, \\
& R_{1313}^{t}=R_{2323}^{t}=\partial_{[3]} Q-Q^{2}, \quad R_{1213}^{t}=2 e A_{[1]} Q, R_{1232}^{t}=-2 e A_{[2]} Q, R_{1323}^{t}=4 e A_{[3]} Q,
\end{aligned}
$$

where $F_{B C}=e_{A}^{\mu} e_{C}^{v} F_{\mu v}=\partial_{B} A_{C}-\partial_{C} A_{B}+\left(\omega_{B C}^{D}-\omega_{C B}^{D}\right) A_{D}$ coincide, by appearance, with the tetrad components of the electromagnetic field tensor rewritten in the basis $\boldsymbol{e}_{A}$. It should be remembered that all the $A_{C}$ here came from the components of the Ricci coefficients of rotation (5.38).

3. The hypersurface $S_{(\mathbf{1 2 0})}$ represents the surface of a given "radius" at all times. It has two spacelike and one timelike tangent vectors $\boldsymbol{V}_{P},(P=0,1,2)$, and a single spacelike normal vector $\boldsymbol{V}_{3}$. The coefficients of the second fundamental form are $L_{12}^{3}=L_{20}^{3}=L_{30}^{3}=0$ and $L_{11}^{3}=L_{22}^{3}=-L_{00}^{3}=\partial_{[3]} \ln \mathcal{R}=Q$. The second fundamental form, $\| I^{3}=L_{P Q}^{3} \mathrm{~d} S^{P} \mathrm{~d} S^{Q}$, is proportional to the first fundamental form $I_{(120)}=\eta_{P Q} \mathrm{~d} S^{P} \mathrm{~d} S^{Q}=\left(\mathrm{d} S^{0}\right)^{2}-$ $\left(\mathrm{d} S^{1}\right)^{2}-\left(\mathrm{d} S^{2}\right)^{2}$ of the $S_{(120)}$,

$$
\|^{3}=-Q \cdot\left[\left(\mathrm{d} S^{0}\right)^{2}-\left(\mathrm{d} S^{1}\right)^{2}-\left(\mathrm{d} S^{2}\right)^{2}\right]=-Q \cdot \mathrm{I}_{(120)} \cdot
$$

Therefore, the hypersurface $S_{(120)}$ is also a totally umbilical submanifold with the mean curvature $H=-Q=m \mathcal{P} / \mathcal{R}>0$. By virtue of Equations (6.2), the vector of (mean) geodesic curvature $H$ is constant and parallel throughout every hypersurface $S_{(120)}$.

The intrinsic part of the Riemann curvature of the hypersurface $S_{(120)}$ has only the following components,

$$
R_{1212}^{t}=2 e\left(\partial_{[1]} A_{[2]}-\partial_{[2]} A_{[1]}\right)-4 e^{2}\left(A_{[1]}^{2}+A_{[2]}^{2}\right)=2 e F_{12},
$$

identical with those of $S_{(12)}$. The extrinsic parts are due to $\omega_{131}=\omega_{232}=-\omega_{030}=-Q$, i.e., the connections that contain normal component $\boldsymbol{e}_{3}$,

$$
R_{1212}^{n}=-R_{1010}^{n}=-R_{2020}^{n}=Q^{2}=m^{2} \sin ^{2} \mathcal{Y} .
$$

Since congruences $\boldsymbol{e}_{0}, \boldsymbol{e}_{1}$ and $\boldsymbol{e}_{2}$ are canonical with respect to the normal congruence $\boldsymbol{e}_{3}$, their lines are the lines of curvature of the hypersurface $S_{(120)}$. If at some point of $S_{(120)}$ we have $\mathrm{II}^{3}=L_{P Q}^{3} \mathrm{~d} S^{P} \mathrm{~d} S^{Q}=0$, then the directions of $\boldsymbol{e}_{0}, \boldsymbol{e}_{1}$ and $\boldsymbol{e}_{2}$ become the asymptotic directions.

4. Surface $S_{(12)}$ is the surface of a given "radius" at a given time and can be viewed as a hypersurface of either $S_{(123)}$ or $S_{(120)}$ with the normals $\boldsymbol{e}_{3}$ or $\boldsymbol{e}_{0}$, respectively. It has two spacelike tangent vectors $\boldsymbol{V}_{P},(P=1,2)$, and two normal vectors $\boldsymbol{V}_{A},(A=0,3)$, timelike $\boldsymbol{V}_{0}$ and spacelike $\boldsymbol{V}_{3}$. Accordingly, there are two second fundamental forms, $\| I^{0}=L_{P Q}^{0} \mathrm{~d} S^{P} \mathrm{~d} S^{Q}$ and $\| I^{3}=L_{P Q}^{3} \mathrm{~d} S^{P} \mathrm{~d} S^{Q}$, with the following coefficients $L_{12}^{0}=L_{12}^{3}=0$, $L_{11}^{0}=L_{22}^{0}=0, \quad L_{11}^{3}=L_{22}^{3}=\partial_{[3]} \ln \mathcal{R}=Q$. The first fundamental form of $S_{(12)}$ is $I_{(12)}=\eta_{P Q} \mathrm{~d} S^{P} \mathrm{~d} S^{Q}=-\left(\mathrm{d} S^{1}\right)^{2}$ $-\left(\mathrm{d} S^{2}\right)^{2}$, and the two second fundamental forms are

$$
\left\|I^{0}=0, \quad\right\|^{3}=-2 Q \cdot I_{(12)} .
$$

Therefore, the 2-d surface $S_{(12)}$ is a totally umbilical submanifold with the mean curvature $H=m \mathcal{P} / \mathcal{R}$ $=m \sin \mathcal{Y}>0$, which is determined by the Dirac field within principal manifold $\mathbb{M}$. The Gaussian curvature $K=\sqrt{k_{1} k_{2}}=m \sin \mathcal{Y}$ is positive. Such a surface can only be the sphere with the radius of curvature $\kappa=1 / H$ [19] [20]. (It is a plane, when $\kappa=0$, but then $\mathcal{R}$ must be uniform and $\mathcal{P}=0$. Here, the spherical shape is a dynamic symmetry since it originates from equations of motion.). Nearly the most important property of submanifolds $S_{(12)}$ follows from the compatibility conditions (5.29) and Equation (6.2), which indicate that the invariant densities $\mathcal{R}, \mathcal{S}, \cdots$ are constant along every 2-d surface $\tau=\tau^{*}=$ const , $\rho=\rho^{*}=$ const. The mean curvature $H$ is constant along $S_{(12)}$ as well. The normal connection for this submanifold can be only due to the components $\omega_{01}^{3}$ and $\omega_{02}^{3}$ of the connection $\omega_{A R}^{B}$, but these vanish identically, $D_{03 ; 1}=D_{03 ; 2}=0$, so that both normal vector fields (and the mean curvature vector) are parallel with respect to the tangent displacements along $S_{(12)}, D_{R} H=0$. The Riemann curvature of $S_{(12)}$ has only one component, $R_{1212}$ and it can be decomposed in two parts. The intrinsic one, $R_{1212}^{t}$, is given by the terms of (6.12) with all indices in tangent space of $S_{(12)}$. The only nonzero connections here are $\omega_{212}=-2 e A_{[2]}$ and $\omega_{121}=2 e A_{[1]}$, so that sectional curvature 
of the $S_{(12)}$,

$$
R_{1212}^{t}=2 e\left(\partial_{[1]} A_{[2]}-\partial_{[2]} A_{[1]}\right)-4 e^{2}\left(A_{[1]}^{2}+A_{[2]}^{2}\right)=2 e F_{12},
$$

is entirely due to the tangent tetrad components of the electromagnetic field $A_{\mu}$. The extrinsic part, $R_{1212}^{n}$, is due to the connections $\omega_{131}=\omega_{232}=-Q$ from the second fundamental form and

$$
R_{1212}^{n}=L_{11}^{0} L_{22}^{0}-L_{11}^{3} L_{22}^{3}=-\left(\partial_{[3]} \ln \mathcal{R}\right)^{2}=-Q^{2} .
$$

5. The surface $S_{(03)}$ represents a given "angular direction" at all "radial" distances and at all times. It has one spacelike and one timelike tangent vectors $\boldsymbol{V}_{P},(P=0,3)$, and two spacelike normal vectors $\boldsymbol{V}_{A},(A=1,2)$. Here, we also have two second fundamental forms, $\|^{1}=L_{P Q}^{1} \mathrm{~d} S^{P} \mathrm{~d} S^{Q}$ and $\|^{2}=L_{P Q}^{2} \mathrm{~d} S^{P} \mathrm{~d} S^{Q}$, with the following coefficients $L_{03}^{1}=L_{03}^{2}=0, L_{00}^{1}=-L_{33}^{1}=0, L_{00}^{2}=-L_{33}^{2}=0$. The first fundamental form of the $S_{(03)}$ is $\mathrm{I}_{03}=\left(\mathrm{d} S^{0}\right)^{2}-\left(\mathrm{d} S^{3}\right)^{2}$ and both second fundamental forms are just zero, $\mathbb{I}^{1}=\mathbb{I}^{2}=0$.

The submanifold $S_{(03)}$ is totally umbilical with the mean curvature $H=0$, and as such is a totally geodesic submanifold. The shape form of $S_{(03)}$ is zero. The normal connection for the coordinate surface $S_{(03)}$ (and only for this surface) does not vanish,

$$
\nabla_{R} \boldsymbol{e}_{1}=-2 e A_{R} \boldsymbol{e}_{2}, \nabla_{R} \boldsymbol{e}_{2}=2 e A_{R} \boldsymbol{e}_{1}, R=0,3,
$$

solely due to the external potential $A_{\mu}, D_{12 ; 0}=-2 e A_{[0]}, D_{12 ; 3}=-2 e A_{[3]}$. A displacement in the directions of $\boldsymbol{e}_{0}$ and $\boldsymbol{e}_{3}$, rotates the tetrad in plane (12). The Riemannian sectional curvature of the $S_{(03)}$ is induced by an ambient space,

$$
R_{0303}^{t}=-\partial_{[3]} Q+Q^{2}
$$

\subsection{Coordinate Lines}

According to Equation (6.2), system (6.5) of PDEs admits, along with the first integrals $\tau(x)=\tau^{*}$ and $\rho(x)=\rho^{*}$ of hypersurfaces $S_{(123)}$ and $S_{(120)}$, respectively, the first integrals $\vartheta(x)=\mathcal{R}(x)=c_{R}, \vartheta(x)=$ $\mathcal{P}(x)=c_{P}$ and $\vartheta(x)=\mathcal{S}(x)=c_{S}$, which must be functions of the former ones, and vice versa,

$$
\mathcal{S}(\mathrm{P})=\mathcal{S}(\rho, \tau), \mathcal{P}(\mathrm{P})=\mathcal{P}(\rho, \tau) \text {, and } \tau=\tau(\mathcal{S}, \mathcal{P}), \rho=\rho(\mathcal{S}, \mathcal{P})
$$

being, ultimately, the known functions of the Dirac field $\psi(\mathrm{P})$. Potentially, one can obtain the functions $\tau$ and $\rho$ purely algebraically,without even solving system (6.5) of PDEs. Every 2-d surface $S_{(12)}$ is fixed not only by the constants $\tau^{*}$ and $\rho^{*}$, but also, e.g., by $C_{S}$ and $C_{P}$, which indicates that surface $S_{(12)}$ belongs to the principal manifold $\mathbb{M}$ without any reference to a coordinate $\mathbb{R}^{4}$. These observations are compli- mentary to the main idea of this work that Dirac field naturally determines the moving frame. Here, the two scalars, e.g., $\mathcal{S}$ and $\mathcal{P}$, can replace the coordinates $\tau$ and $\rho$ (similarly to the hodograph transformation in hydrodynamics). From Equation (6.2) with tetrad index $A=0$ one can see that neither of the scalars $\mathcal{S}, \mathcal{P}, \mathcal{R}$ depends on the time variable $\tau$ (or $S^{0}$ ). Therefore, these quantities depend only on the radial variable $\rho$ (or, equivalently, on the affine parameter $\sigma=S^{3}$ ).

1. Radial lines. When a geodesic line is given in the parametric form, $x^{\mu}=x^{\mu}(\sigma)$, the unit tangent vector is $e_{[3]}^{\mu}=\mathrm{d} x^{\mu} / \mathrm{d} \sigma$. The affine parameter of the radial geodesic lines is $\sigma=S^{3}$, but it differs from the parameter $\rho$ of the hypersurfaces $\rho(x)=\rho^{*}=$ const, which determines distance (5.21) at some moment of the world time $\tau$ (5.12). In terms of the variable $\sigma$, the ODE for geodesic line with the tangent vector $e_{[3]}^{\mu}$ is

$$
e_{[3]}^{\mu} \nabla_{\mu} e_{[3]}^{\lambda}=e_{A}^{\lambda} \omega_{33}^{A}=0=\frac{\mathrm{d} x^{\mu}}{\mathrm{d} \sigma} \nabla_{\mu}\left(\frac{\mathrm{d} x^{\lambda}}{\mathrm{d} \sigma}\right)=\frac{\mathrm{d}^{2} x^{\lambda}}{\mathrm{d} \sigma^{2}}+\Gamma_{\nu \mu}^{\lambda} \frac{\mathrm{d} x^{\nu}}{\mathrm{d} \sigma} \frac{\mathrm{d} x^{\mu}}{\mathrm{d} \sigma},
$$

where the connection $\Gamma_{v \mu}^{\lambda}$ is defined by Equation (3.14). The ODE for a geodesic line $x^{\lambda}(\rho)$ in terms of the physical variable $\rho$ that can be obtained by means of a simple transformation, $\mathrm{d} x^{\mu} / \mathrm{d} \sigma=\mathcal{R} \mathrm{d} x^{\mu} / \mathrm{d} \rho$, and reads as

$$
\frac{\mathrm{d}^{2} x^{\lambda}}{\mathrm{d} \sigma^{2}}+\Gamma_{v \mu}^{\lambda} \frac{\mathrm{d} x^{\nu}}{\mathrm{d} \sigma} \frac{\mathrm{d} x^{\mu}}{\mathrm{d} \sigma}=-\frac{\mathrm{d} \ln \mathcal{R}}{\mathrm{d} \rho} \frac{\mathrm{d} x^{\lambda}}{\mathrm{d} \rho}=\frac{m \mathcal{P}}{\mathcal{R}^{2}} \frac{\mathrm{d} x^{\lambda}}{\mathrm{d} \rho}=\frac{m \mathcal{P}}{\mathcal{R}} e_{[3]}^{\lambda},
$$

where the r.h.s. does not contain derivatives of the Dirac field and it clearly manifests that the (not unit) tangent 
vector $\mathrm{d} x^{\lambda} / \mathrm{d} \rho$ and its change are parallel along the "radial" geodesic curve.

2. The lines of the world time. The acceleration of the unit tangent vector of the lines of the vector current $j^{\mu}$ is

$$
e_{[0]}^{\mu} \nabla_{\mu} e_{[0]}^{\lambda}=e_{A}^{\lambda} \omega_{00}^{A}=e_{[3]}^{\lambda} \omega_{00}^{3}=m(\mathcal{P} / \mathcal{R}) e_{[3]}^{\lambda},
$$

and it has only the radial component (precisely the same as radial geodesic (6.25)), which equals in magnitude but has opposite sign with respect to the mean curvature vector of surface $S_{(12)}$ and hypersurface $S_{(120)}$. The ODE for the trajectory $x^{\lambda}(\tau)$ reads as

$$
\frac{\mathrm{d}^{2} x^{\lambda}}{\mathrm{d} \tau^{2}}+\Gamma_{v \mu}^{\lambda} \frac{\mathrm{d} x^{\nu}}{\mathrm{d} \tau} \frac{\mathrm{d} x^{\mu}}{\mathrm{d} \tau}=m \mathcal{P} \frac{\mathrm{d} x^{\lambda}}{\mathrm{d} \rho}=\frac{m \mathcal{P}}{\mathcal{R}} e_{[3]}^{\lambda} .
$$

Obviously, the line of the vector current that passes through a point with the radial coordinate $\rho^{*}$ never leaves the the surface $\rho=\rho^{*}=$ const. Therefore, there is no flux of the charge density $\mathcal{R}$ in the outside direction, which is an indirect but indisputable evidence of localization.

3. The coordinate net over $\boldsymbol{S}_{(12)}$. Finally, the lines of the Dirac currents $\Theta^{\mu}$ and $\Phi^{\mu}$ are also bound to the surface $\rho=\rho^{*}=$ const . Indeed, for the curves $x^{\lambda}=x^{\lambda}\left(S^{1}\right)$ and $x^{\lambda}=x^{\lambda}\left(S^{2}\right)$ we have

$$
\begin{aligned}
& e_{[1]}^{\mu} \nabla_{\mu} e_{[1]}^{\lambda}=e_{A}^{\lambda} \omega_{11}^{A}=e_{[3]}^{\lambda} \omega_{11}^{3}+e_{2}^{\lambda} \omega_{11}^{2}=-m(\mathcal{P} / \mathcal{R}) e_{[3]}^{\lambda}-2 e A_{[1]} e_{[[2]]}^{\lambda}, \\
& e_{[2]}^{\mu} \nabla_{\mu} e_{[2]}^{\lambda}=e_{A}^{\lambda} \omega_{22}^{A}=e_{[3]}^{\lambda} \omega_{22}^{3}+e_{[1]}^{\lambda} \omega_{22}^{1}=-m(\mathcal{P} / \mathcal{R}) e_{[3]}^{\lambda}+2 e A_{[2]} e_{[1]}^{\lambda},
\end{aligned}
$$

so that they have the same normal component of the mean curvature vector, and they are bent within surface $S_{(12)}$ even when the components $A_{I}=e_{I}^{\mu} A_{\mu} \neq 0, I=1,2$.

To summarize, all the currents passing in a tangent direction through a point on hypersurface $S_{(120)}$ of a given radius $\rho^{*}$ never leave this surface.

\section{Conclusions}

The (hyper)surfaces emerging from the Dirac equation and differential identities for the Dirac currents point to a fairly simple geometric structure of the lines and surfaces of the admissible coordinate net. These surfaces are built into the Dirac matter and completely determined by the latter. We will extensively refer to their properties in the second part [8] of this work. They will be used to write down the exact nonlinear Dirac equations and to find their analytic solutions, which represent a finite-sized stable particle. These solutions will necessarily be localized and have a spherical symmetry. This symmetry is not contemplated as a property of the ambient space. Within the framework of the matter-induced affine geometry, the spherical symmetry is the property of a solution, and thus is a dynamic symmetry. [8].

A general discussion of the method, its results and perspectives is postponed till the last section of the Ref.

\section{References}

[1] Makhlin, A. (2001) Physical Review C, 64, Article ID: 064904. http://dx.doi.org/10.1103/PhysRevC.64.064904

[2] Sakharov, A.D. (1967) JETP Letters, 5, 24-27.

[3] Dolgov, A.D. (2007) Cosmological Charge Asymmetry and Rare Processes in Particle Physics. Les Rencontres de Physique de La Vallee d'Aoste, 4-10 March 2007, Aosta Valley, Italy, 5 p. arXiv:0706.1229 [hep-ph]

[4] Dolgov, A.D. (2010) Physics of Atomic Nuclei, 73, 588-592. http://dx.doi.org/10.1134/S1063778810040022

[5] Dolgov, A.D. (2015) Antimatter in the Universe and Laboratory. The European Physical Journal Conferences, 95, Article ID: 03007.

[6] Serpico, P.D. (2012) Astroparticle Physics, 39-40, 2-11. http://dx.doi.org/10.1016/j.astropartphys.2011.08.007

[7] Makhlin, A. (2010) Localization, CP-Symmetry and Neutrino Signals of the Dirac Matter. arxiv:1005.2693 [math-ph]

[8] Makhlin, A. (2016) Journal of Modern Physics, 7, 662-679. http://dx.doi.org/10.4236/jmp.2016.77066

[9] Takabayasi, T. (1958) Il Nuovo Cimento (1955-1965), 7, 118-121. http://dx.doi.org/10.1007/BF02746891

[10] Takahashi, Y. (1983) Journal of Mathematical Physics, 24, 1783. http://dx.doi.org/10.1063/1.525896 
[11] Takahashi, Y. (1982) Physical Review D, 26, 2169. http://dx.doi.org/10.1103/PhysRevD.26.2169 Crawford, J.P. (1985) Journal of Mathematical Physics, 26, 1439. http://dx.doi.org/10.1063/1.526906

[12] Israel, W. and Nester, J.M. (1981) Physics Letters A, 81, 259. http://dx.doi.org/10.1016/0375-9601(81)90951-8

[13] Cartan, E. (1966) The Theory of Spinors. Hermann, Paris.

[14] Ne’eman, Y. (1978) Annales de l'Institut Henri Poincaré, Section A, 28, 369.

Hehl, F.W, Lord, E.A. and Ne’eman, Y. (1978) Physical Review D, 17, 428. http://dx.doi.org/10.1103/PhysRevD.17.428

[15] Cartan, E. (2001) Riemannian Geometry in an Orthogonal Frame. World Scientific, Singapore.

[16] Fock, V. (1929) Zeitschrift für Physik, 57, 261-277. http://dx.doi.org/10.1007/BF01339714

[17] Eisenhart, L.P. (1926) Riemannian Geometry. Princeton University Press, Princeton.

[18] Levi-Civita, T. (1926) The Absolute Differential Calculus. Blackie \& Son Ltd., London and Glasgow. (Dover, 1977)

[19] Stoker, J.J. (1969) Differential Geometry. Wiley Interscience, Hoboken.

[20] O’Neill, B. (1966) Elementary Differential Geometry. Academic Press, Cambridge, Massachusetts. 